\title{
A new formulation of a Hénon-Heiles potential with additional singular gravitational terms
}

\author{
Euaggelos E. Zotos \\ Department of Physics, School of Science, \\ Aristotle University of Thessaloniki, \\ GR-54124, Thessaloniki, Greece \\ Corresponding author's email: evzotos@physics.auth.gr \\ Wei Chen \\ LMIB \& School of Mathematics and Systems Science, \\ Beihang University, Beijing 100191, China \\ Peng Cheng Laboratory, Shenzhen, Guangdong, \\ China, 518055 \\ Beijing Advanced Innovation Center for Big Data and Brain Computing, \\ Beihang University, Beijing 100191, China \\ Juan F. Navarro \\ Department of Applied Mathematics, \\ University of Alicante, Carretera San Vicente del Raspeig s/n, \\ 03690, Alicante, Spain \\ Tareq Saeed \\ Nonlinear Analysis and Applied Mathematics (NAAM)-Research Group, \\ Department of Mathematics, Faculty of Science, \\ King Abdulaziz University, P.O. Box 80203, Jeddah \\ 21589, Saudi Arabia \\ Received (to be inserted by publisher)
}

\begin{abstract}
We examine the orbital dynamics in a new Hénon-Heiles system with an additional gravitational potential, by classifying sets of starting conditions of trajectories. Specifically, we obtain results on how the total orbital energy along with the transition parameter influence the overall dynamics of the massless test particle, as well as the respective time of escape/collision. By using modern diagrams with color codes we manage to present the different types of basins of the system. We show that the character of the orbits is highly dependent on the energy and the transition parameter.
\end{abstract}

Keywords: Hénon-Heiles system - Chaos - Fractals

\section{Introduction}

The Hénon-Heiles system was initially used for modeling how stars move around a galactic center and constitutes a paradigm for nonlinear dynamics of Hamiltonian systems. This problem has attracted the 
activity of an extensive number of works (see e.g., [Aguirre et al., 2001; Aguirre \& Sanjuán, 2003; Aguirre et al., 2003, 2009; Barrio et al., 2008; Blesa et al., 2012; Brack, 2001; Cjang et al., 1982; Churchill et al., 1975; Coccolo et al., 2013; Conte et al., 2005; De Moura \& Letelier, 1999; Deprit \& Henrard, 1968, 1969; Fordy, 1991; Hénon \& Heiles, 1964; Innanen, 1985; Ozaki \& Kurosaki, 1996; Poincaré, 1892; Zotos, 2015, 2017a]). The interest in this system was initially motivated by the research of some additional conservation laws in certain galactic potentials presenting an axis of symmetry. In their pioneer work, Hénon and Heiles [Hénon \& Heiles, 1964] investigated the problem according to which a third integral of motion exists in axisymmetric potentials. Moreover, it was shown that this isolated integral is present only for a limited set of starting conditions, through a numerical exploration of the system.

As we know, the Hénon-Heiles Hamiltonian has a transition to chaos at a critical value of the energy and is strongly chaotic for large energies. For small values of the energy, the system appears to be integrable. However, as the energy increases, some small chaotic regions arise and, when the energy is equal to the critical escape energy the chaotic regions dominate the configuration space. In this system, when the energy is below the escape value the curves of zero velocity are closed, which implies that only bounded motion is possible. On the other hand, for energies above the escape level, the limiting curves open and three exit channels appear, which lead to escape to infinity.

In [Aguirre et al., 2001], the authors analyzed the final state of the test particles showing the high unpredictability of the system. In particular, it was proved that the escape basins do not have only fractal boundaries but they also obey the so-called Wada property (when any point of the boundary belongs simultaneously to the boundaries of at least two more basins). Recently, in [Zotos, 2015, 2017a] we studied in detail the influence of the energy on the different types of motion, by monitoring how the energy affects the percentages of the different types of trajectories.

Over the past few years, the use of pseudo or post-Newtonian dynamics has become very popular (see e.g., [Dubeibe et al., 2017a,b; Suraj et al., 2019; Zotos, 2017b; Zotos et al., 2018, 2019]). This is because the additional gravitational terms allow us to model properties and predict phenomena which are not possible by using the classical Newtonian dynamics. In this work, we will introduce a new version of the HénonHeiles system with extra gravitational terms. These terms will give us the opportunity to realistically model the orbital dynamics of a test particle moving in the central parts of a galaxy, where a black hole is usually present, thus creating a singularity that cannot be modeled by the classical Hénon-Heiles problem.

The article is organized according to the following plan: The dynamical properties of the new modelpotential are described in Section 2. Section 3 contains the main numerical results of our investigation, regarding the classification of the orbits, while in Section 4 the most important conclusions are emphasized.

\section{The Hamiltonian system}

The classical Hénon-Heiles potential is given by

$$
V_{h h}(x, y)=\frac{1}{2}\left(x^{2}+y^{2}\right)+x^{2} y-\frac{1}{3} y^{3}
$$

and it can describe the local motion of stars, moving in the central regions of galaxies.

However, today we know that black holes reside at the centers of almost all galaxies. Unfortunately, the simple polynomial potential of Eq. (1) can not take into account the presence of a gravitational singularity at the center of the galaxy. On this basis, we decided to expand the classical potential by adding appropriate gravitational terms. The easiest way to model the properties of a gravitational singularity is by using a Plummer potential [Plummer, 1991]. Then, the new modified Hénon-Heiles potential reads

$$
V(x, y)=V_{h h}(x, y)-G M\left(\frac{1}{r}+\frac{\epsilon}{r^{3}}\right),
$$

where of course $r=\sqrt{x^{2}+y^{2}}$, while $G$ is the gravity constant and $M$ is the black hole's mass. For simplicity reasons we may set $G M=1$.

The quantity $\epsilon$ is a physical transition parameter that controls the ratio between the classical and the stronger Newtonian gravity. The values of $\epsilon$ lie in the interval $[0,1]$. Form Eq. (2) it becomes evident that when $\epsilon=0$ we have the classical Newtonian gravity of the form $1 / r$. On the other hand, when $\epsilon>0$ an 
additional gravitational field, of the form $1 / r^{3}$, is created, which is of course stronger than the Newtonian one. At this point, it should be stressed out that terms of the form $1 / r^{3}$ are very common in general relativity, for describing gravity stronger than the classical Newtonian. Even the simplest Schwarzschild effective potential contains such a term [Schwarschild, 1916]. Therefore, the new modified potential can realistically describe the motion of massless particles in the central parts of galaxies and in particular near the black hole.

The motion of the massless particle on the plane $(x, y)$ follows the set of equations

$$
\begin{aligned}
& \ddot{x}=-\frac{\partial V}{\partial x}=-x\left[1+2 y+G M\left(\frac{1}{r^{3}}+\frac{3 \epsilon}{r^{5}}\right)\right], \\
& \ddot{y}=-\frac{\partial V}{\partial y}=-y\left[1-y+G M\left(\frac{1}{r^{3}}+\frac{3 \epsilon}{r^{5}}\right)\right]-x^{2} .
\end{aligned}
$$

Similarly, the derivatives of second order of $V(x, y)$ are

$$
\begin{aligned}
& \frac{\partial^{2} V}{\partial x^{2}}=1+2 y+G M\left(\frac{1}{r^{3}}-\frac{3\left(x^{2}+\epsilon\right)}{r^{5}}-\frac{15 \epsilon x^{2}}{r^{7}}\right), \\
& \frac{\partial^{2} V}{\partial x \partial y}=2 x-3 G M x y\left(\frac{1}{r^{5}}+\frac{5 \epsilon}{r^{7}}\right), \\
& \frac{\partial^{2} V}{\partial y \partial x}=\frac{\partial^{2} V}{\partial x \partial y}, \\
& \frac{\partial^{2} V}{\partial y^{2}}=1-2 y+G M\left(\frac{1}{r^{3}}-\frac{3\left(y^{2}+\epsilon\right)}{r^{5}}-\frac{15 \epsilon y^{2}}{r^{7}}\right) .
\end{aligned}
$$

The Hamiltonian of the system is given by

$$
H=\frac{1}{2}\left(\dot{x}^{2}+\dot{y}^{2}\right)+V(x, y)=E,
$$

where as usual $E$ is the conserved value of the energy integral.

In the case of the classical Hénon-Heiles potential, we have four points of equilibrium. In the case where the additional gravitational terms are present the total number of the equilibria reduces to three since the central one, located at $(0,0)$, becomes a singularity. In Fig. 1 we present the location of the points of equilibrium along with the contours of constant potential $V(x, y)$, when $\epsilon=0.5$ and $E=-0.5$. We see that all three libration points belong to the same circle.

As expected the locations of the points of equilibrium depend on the particular value of $\epsilon$. Here, it should be noted that the coordinates of the points of equilibrium cannot be derived in a closed form, due to the existence of the gravitational terms. Nevertheless, we can use numerical techniques (e.g., the Newton-Raphson method) for analytically obtain their locations. Fig. 2(a-c) shows the evolution of the coordinates of the libration points, when $\epsilon \in[0,1]$. In particular, we depict the parametric evolution of the $y$ coordinate of the equilibrium points $L_{1}$, along with the $(x, y)$ coordinates of the equilibrium point $L_{2}$. Note that the libration point $L_{3}$ has the same $y$ coordinate as $L_{2}$, while the $x$ coordinate has an opposite sign.

Using the numerical data, regarding the coordinates $(x, y)$ of the libration points, shown in Fig. 2, we can interpolate them and obtain semi-analytical functions of $\epsilon$. Our analysis indicates that in all cases the coordinates of the points of equilibrium can be sufficiently approximated by a third-order polynomial of the form

$$
f(\epsilon)=a_{0}+a_{1} \epsilon+a_{2} \epsilon^{2}+a_{3} \epsilon^{3} .
$$

In Table 1 we show the exact numerical coefficients for the coordinates of the libration points $L_{1}$ and $L_{2}$.

Knowing the coordinates of the points of equilibrium we can also obtain their linear stability. A point of equilibrium is linearly stable if and only if for the particular coordinates $\left(x_{0}, y_{0}\right)$ the quartic characteristic equation

$$
\lambda^{4}+\left(V_{x x}+V_{y y}\right) \lambda^{2}+V_{x x} V_{y y}-V_{x y}^{2}=0
$$




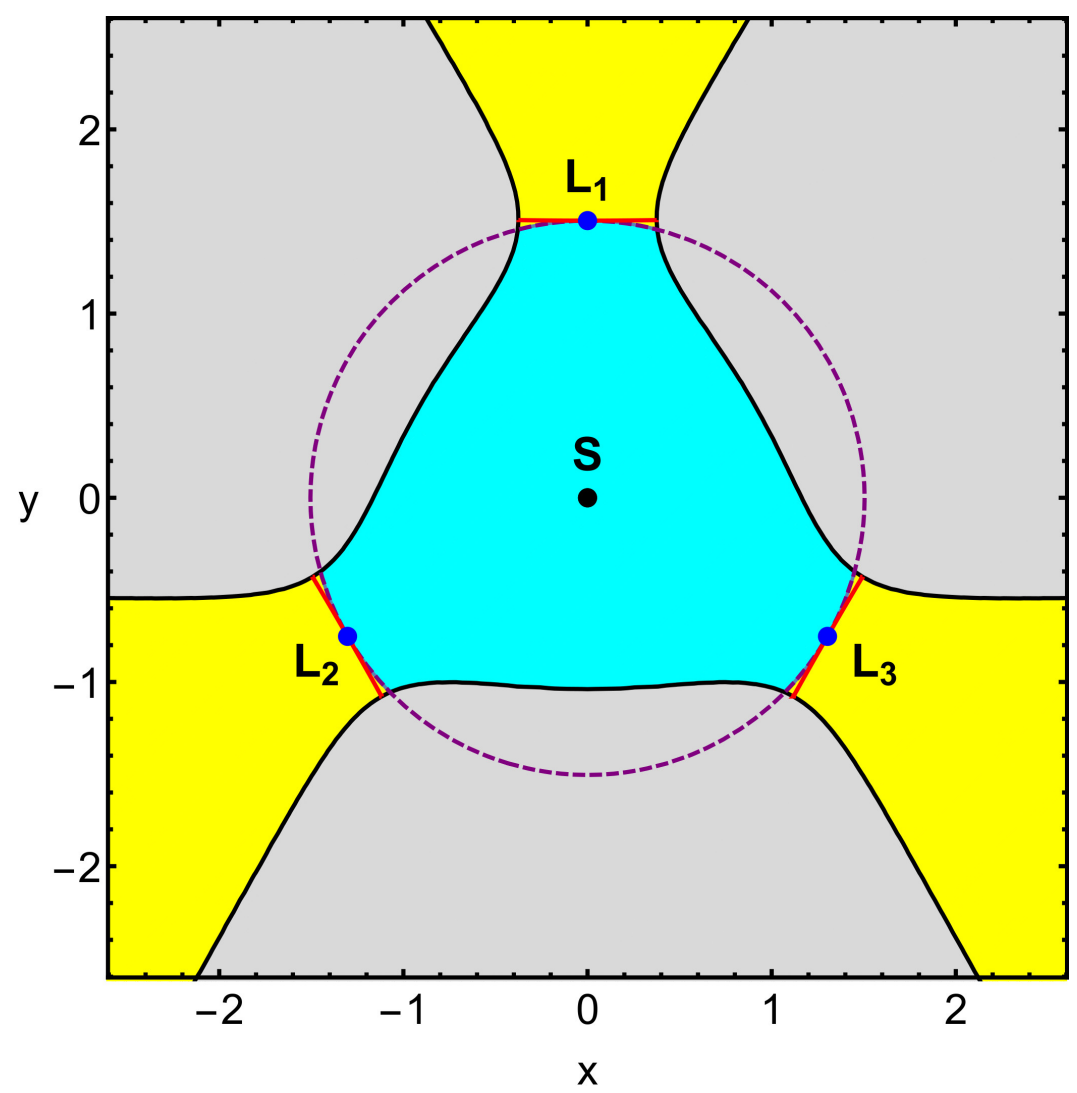

Fig. 1. The contours of the modified Hénon-Heiles potential on the plane $(x, y)$, when $\epsilon=0.5$ and $E=-0.5$. The blue dots indicate the positions of the points of equilibrium $L_{i}, i=1, \ldots, 3$, while the black dot denotes the central singularity $S$. The configuration space is divided into three areas: (i) the energetically forbidden regions (gray), (ii) the interior region (cyan), and (iii) the exterior region (yellow). The red lines correspond to the unstable Lyapunov orbits, which distinguish between the interior and the exterior region of the system. (Color figure online).

(a)

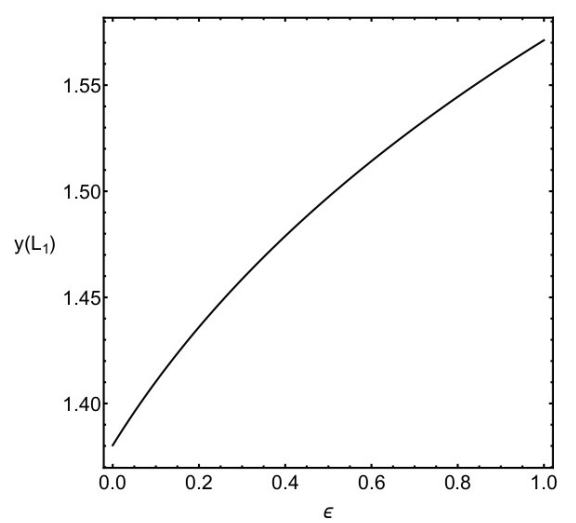

(b)

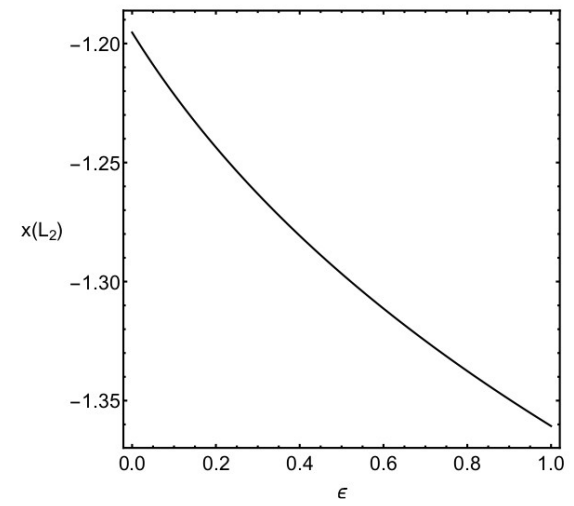

(c)

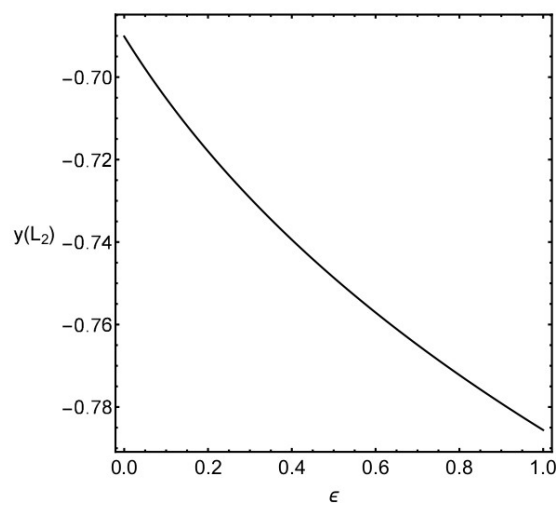

Fig. 2. Evolution of the coordinates of the libration points, as a function of $\epsilon$. (a): $y\left(L_{1}\right) ;(\mathrm{b}): x\left(L_{2}\right) ;(\mathrm{c}): y\left(L_{2}\right)$.

has four pure imaginary roots, where $V_{x x}, V_{y y}$, and $V_{x y}$ are the derivatives of second order of $V(x, y)$. The sufficient and necessary conditions for the existence of four pure imaginary roots are the following

$$
\begin{aligned}
\left(V_{x x}+V_{y y}\right)^{2}-4\left(V_{x x} V_{y y}-V_{x y}^{2}\right) & >0, \\
V_{x x}+V_{y y} & >0, \\
V_{x x} V_{y y}-V_{x y}^{2} & >0,
\end{aligned}
$$


Table 1. The numerical coefficients of the polynomial function $f(\epsilon)$, which approximates the $(x, y)$ coordinates of the points of equilibrium.

\begin{tabular}{cccc}
\hline & $y\left(L_{1}\right)$ & $x\left(L_{2}\right)$ & $y\left(L_{2}\right)$ \\
\hline$a_{0}$ & 1.38125 & -1.19621 & -0.69062 \\
$a_{1}$ & 0.30446 & -0.26367 & -0.15223 \\
$a_{2}$ & -0.17370 & 0.15043 & 0.08685 \\
$a_{3}$ & 0.05972 & -0.05172 & -0.02986 \\
\hline
\end{tabular}
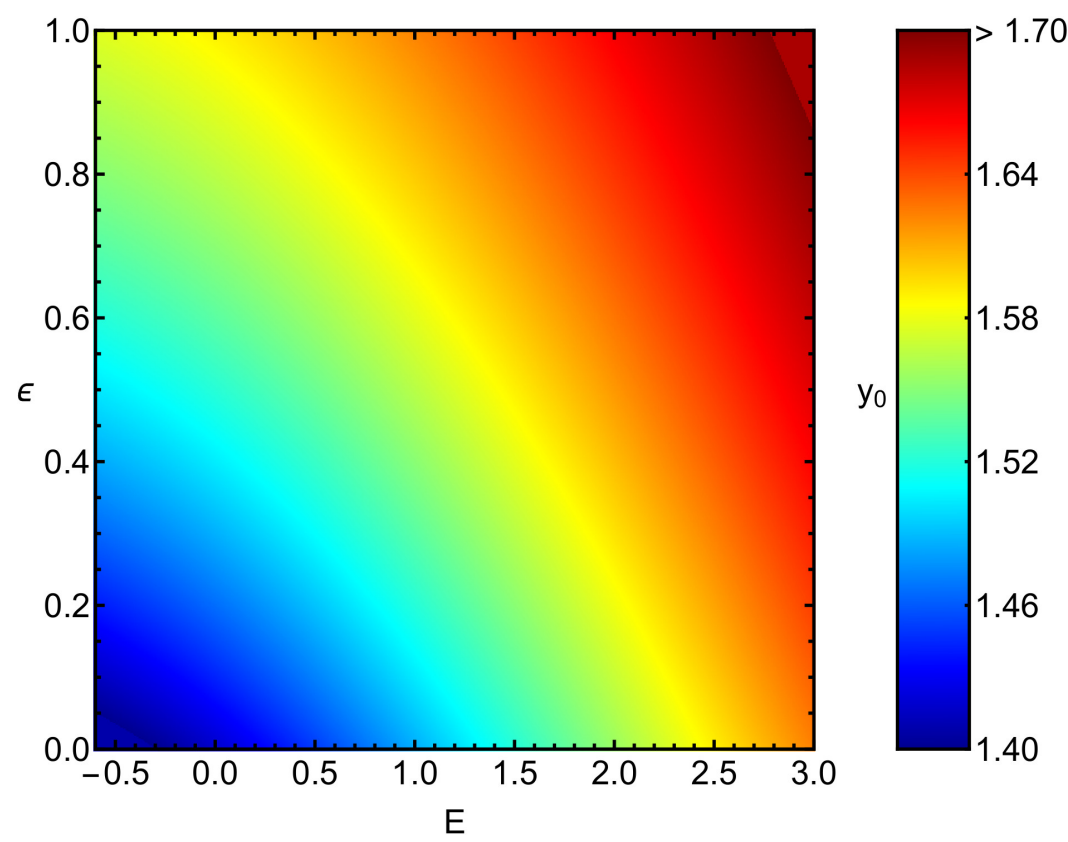

Fig. 3. Evolution of the $y_{0}$ coordinate of the upper Lyapunov orbit, as a function of $E$ and $\epsilon$. (Color figure online).

and must be simultaneously fulfilled.

Our computations suggest that all the points of equilibrium of the system are always linearly unstable, for all possible values of $\epsilon$.

As we know, for energies larger than the energy of escape there is a Lyapunov orbit [Lyapunov, 1907] near the saddle points, at each exit channel. In Fig. 1 we have seen that an unstable Lyapunov orbit passes through each of the equilibrium points. These Lyapunov orbits divide the energetically allowed areas of the configuration $(x, y)$ space into two parts: (i) the interior region (cyan) and (ii) the exterior region (yellow). Let us now consider the initial condition $\left(x_{0}, y_{0}, \dot{x_{0}}, \dot{y}_{0}\right)$ of the upper Lyapunov orbit (corresponding to the point of equilibrium $L_{1}$ ), for given values of the energy $E_{0}$ and the transition parameter $\epsilon_{0}$, with $x_{0}=y_{0}=0$. If the value of the coordinate $y_{0}$ is known, then $\dot{x_{0}}$ can be obtained through the value of the energy. Thus, we can represent the value of $y_{0}$ in terms of the energy and $\epsilon$, for the upper Lyapunov orbit. In Fig. 3, we show, using a color-coded diagram, how the initial condition $y_{0}$ of the upper Lyapunov orbit evolves, as a function of the energy and $\epsilon$. We observe clearly that the value of $y_{0}$ grows with these two parameters. The coordinates $\left(x_{0}, y_{0}\right)$ of the other two Lyapunov orbits can easily be obtained by rotating the coordinates of the upper Lyapunov orbit by $+2 \pi / 3$ and $-2 \pi / 3$.

Once more the data, regarding the $y_{0}$ coordinate of the upper Lyapunov orbit, can be interpolated so as to derive a semi-analytical function of the form $y_{0}(E, \epsilon)$. Our tests suggest that the best fit is a 


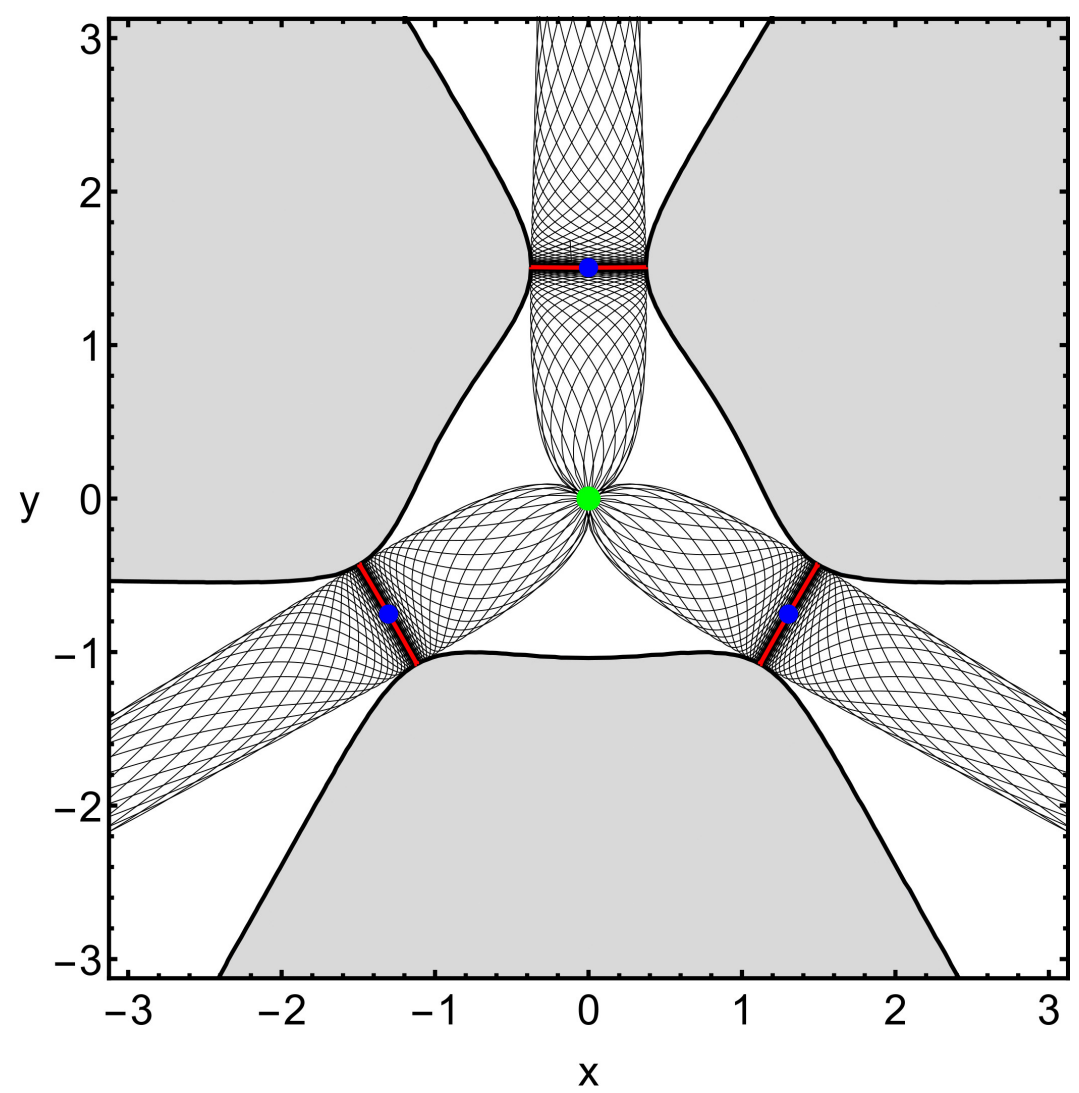

Fig. 4. The structure of the stable manifolds of the Lyapunov orbits (red solid lines), on the plane $(x, y)$. The light green dot indicates the central singularity, while the blue dots pinpoint the positions of the three points of equilibrium. (Color figure online).

fourth-order polynomial function of the form

$$
\begin{aligned}
y_{0}(E, \epsilon) & =a_{0}+b_{1} E+b_{2} E^{2}+b_{3} E^{3}+b_{4} E^{4} \\
& +c_{1} \epsilon+c_{2} \epsilon^{2}+c_{3} \epsilon^{3}+c_{4} \epsilon^{4},
\end{aligned}
$$

where $a_{0}=1.44, b_{1}=0.03871, b_{2}=0.00817, b_{3}=-0.00176, b_{4}=0.00014, c_{1}=0.19741, c_{2}=-0.11110$, $c_{3}=0.07315, c_{4}=-0.02437$.

The stable manifolds associated to the Lyapunov periodic orbits form the local boundaries of the escape channels. In Fig. 4 we show, using a backward integration of a set of starting conditions, the stable manifolds of the Lyapunov orbits, until their intersection with the hyperplane $y=0$ or until they leave the potential well. We observe that all the orbits lead to a collision with the central singularity. It should be emphasized that the structure of the unstable manifolds on the plane $(x, y)$ is completely identical to that of the stable manifolds.

\section{The nature of orbits}

The nature of motion of a massless particle can be revealed by classifying starting conditions of trajectories. The new modified Hénon-Heiles system retains the $2 \pi / 3$ symmetry of the classical Hénon-Heiles system. This symmetry should also be observed in the dynamics of the system. In particular, for values of $E$ higher that the escape energy the three channels of escape should be equiprobable. In other words, the amount of escaping orbits, corresponding to the three channels of escape should be equal. This can only be achieved 
(a)

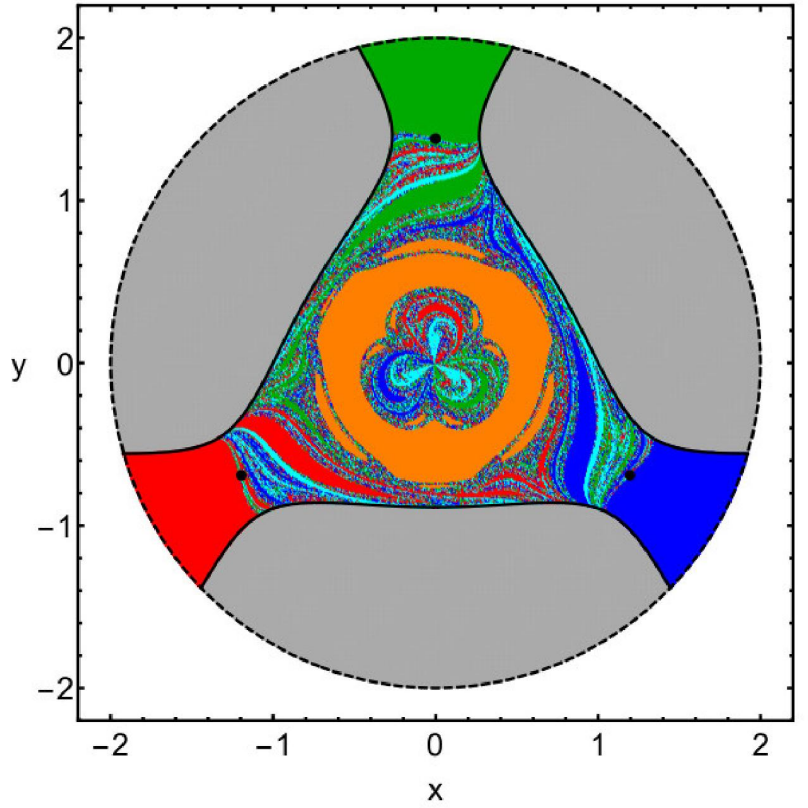

(c)

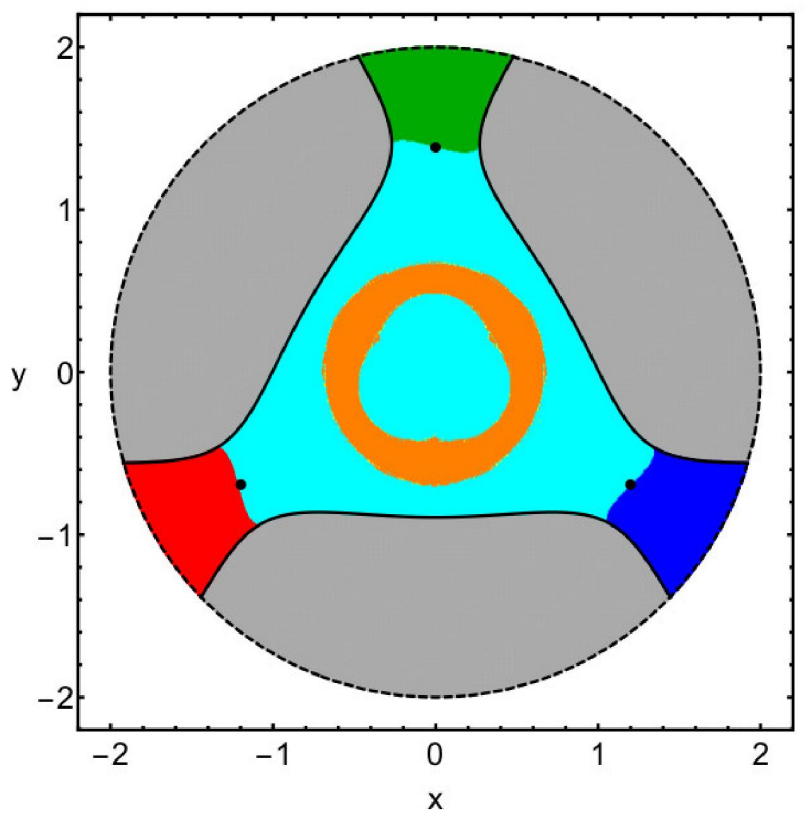

\section{Escape $1 \quad$ Escape 2 Escape 3}

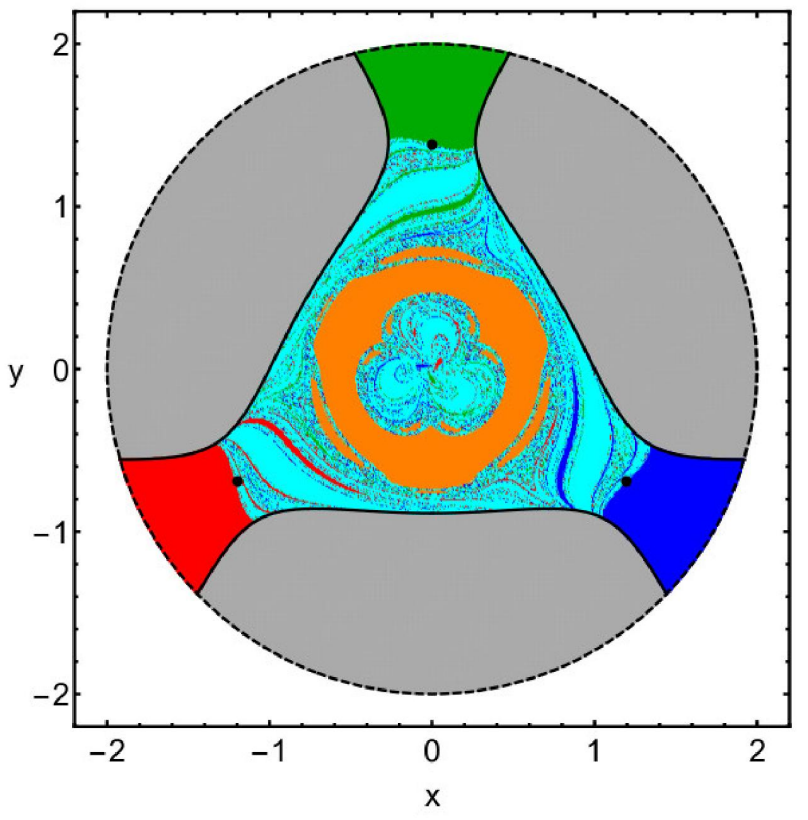

(d)

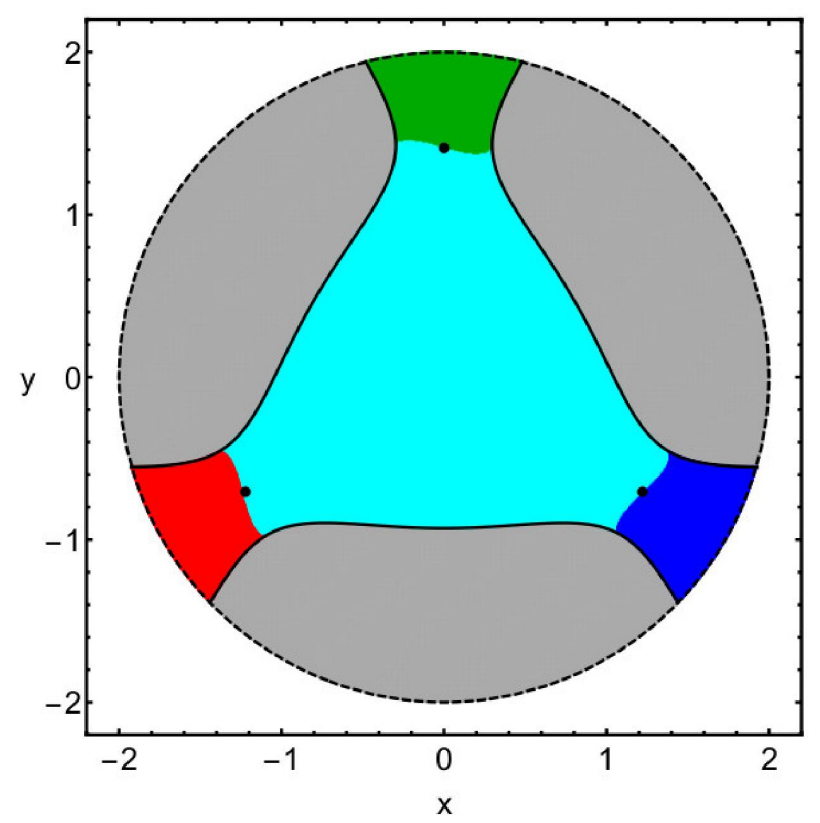

Regular Chaotic Collision

Fig. 5. Basin color diagrams on the plane $(x, y)$, for $E=-0.5$, when (a): $\epsilon=0,(\mathrm{~b}): \epsilon=0.0002,(\mathrm{c}): \epsilon=0.01,(\mathrm{~d}): \epsilon=0.1$. Black dots pinpoint the points of equilibrium. (Color figure online).

when the initial velocities of the test particle are given by

$$
\begin{aligned}
& \dot{x_{0}}=-\frac{y_{0}}{r_{0}} f\left(x_{0}, y_{0}, E_{0}\right), \\
& \dot{y_{0}}=+\frac{x_{0}}{r_{0}} f\left(x_{0}, y_{0}, E_{0}\right),
\end{aligned}
$$


(a)
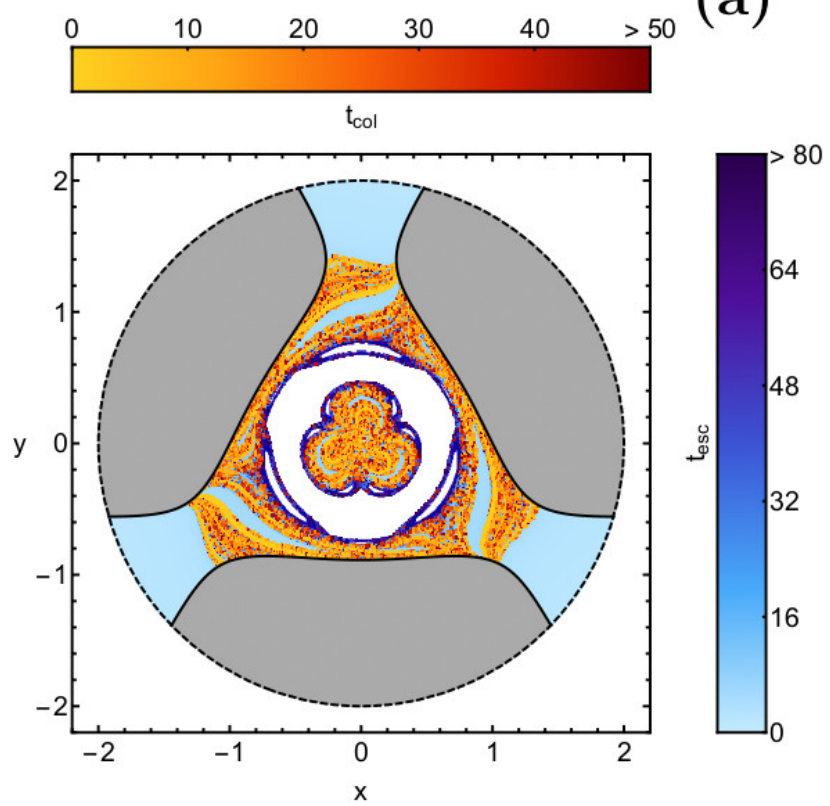

(c)

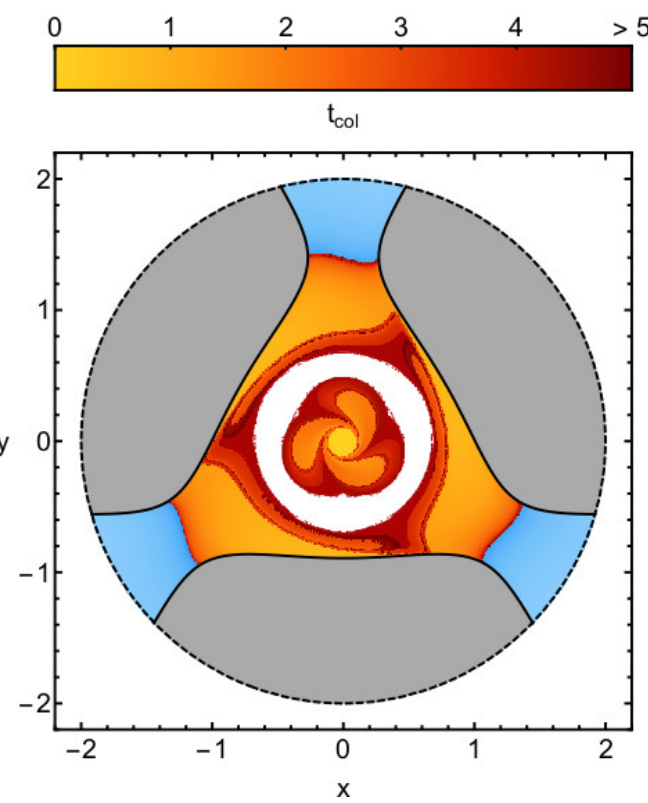

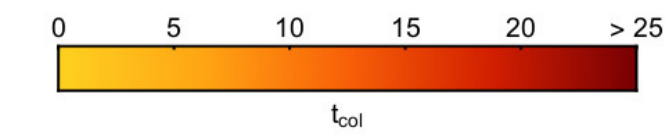

(b)
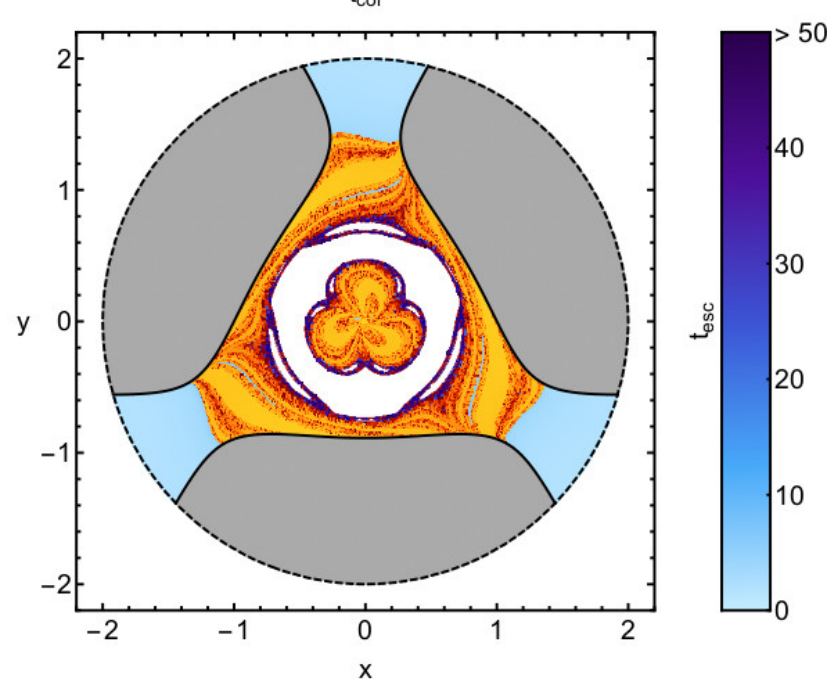

(d)

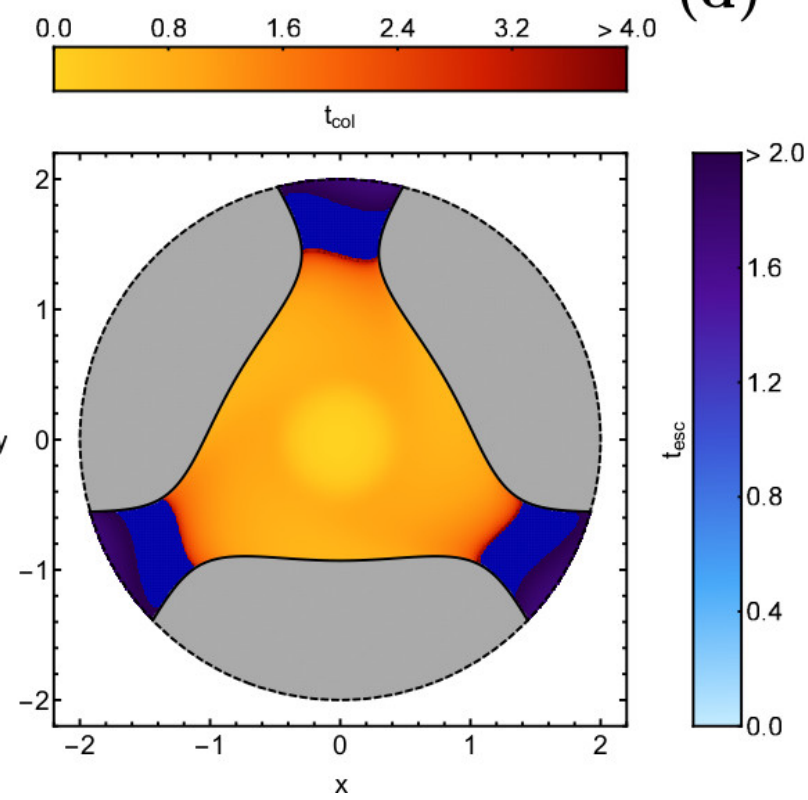

Fig. 6. Distributions of the collision and the escape time of the trajectories, for the initial conditions shown in parts (a)-(d) of Fig. 5. (Color figure online).

where $E_{0}$ is the orbital energy level, $r_{0}=\sqrt{x_{0}^{2}+y_{0}^{2}}$, while $f\left(x_{0}, y_{0}, E_{0}\right)=\sqrt{2\left(V\left(x_{0}, y_{0}\right)-E_{0}\right)}$.

To obtain the orbital dynamics we numerically integrate, in several planes of two dimensions, $1024 \times$ 1024 uniform grids of initial conditions. During the numerical integration, the maximum allowed time of the test particle is limited to $10^{4}$ time units, with a variable time step and the regularization method of Lemaitre, in case the test particle enters a region of radius $R_{\text {reg }}=10^{-2}$, around the central singularity. Our aim is to distinguish between the following types of motion of the massless particle: (i) escaping motion, (ii) collision motion, and (iii) bounded motion. A test particle is considered to escape when it crosses the circle with radius $R=\sqrt{x^{2}+y^{2}}=2$, with velocity pointing outward, while collision motion occurs when the test particle enters the circle with radius $R=10^{-5}$, with velocity pointing inward, thus following a computational methodology and numerical criteria similar to that used in [Nagler, 2004, 2005]. In the case 
(a)

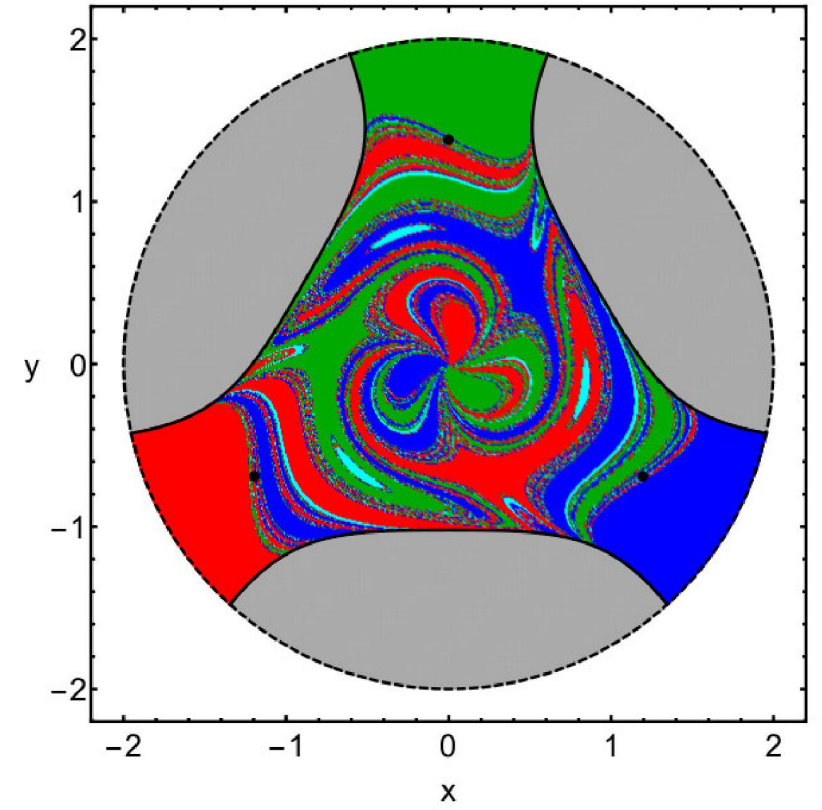

(c)

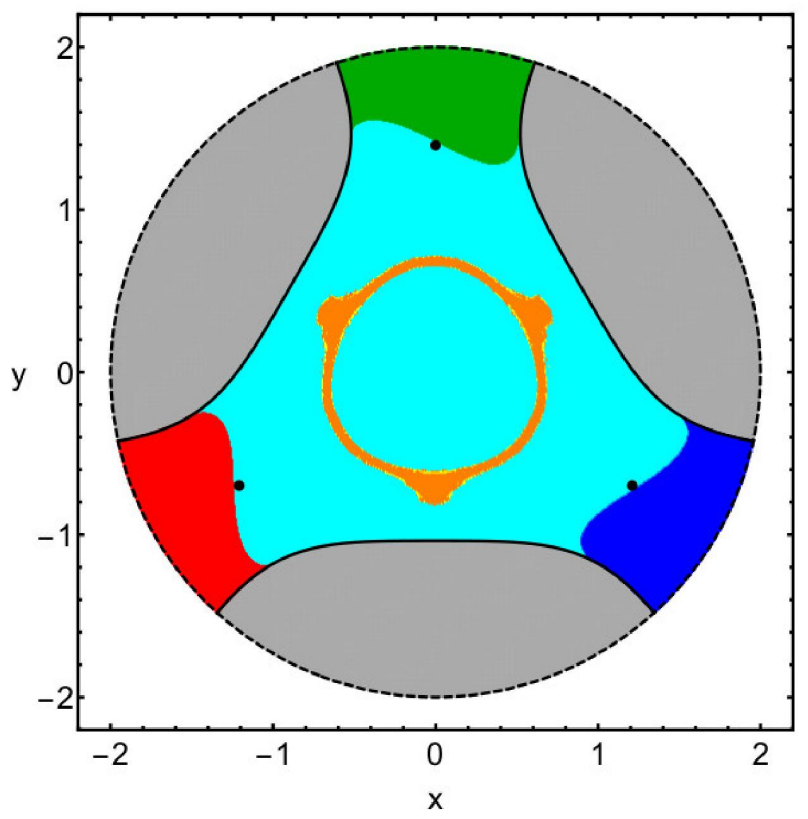

\section{Escape $1 \quad$ Escape $2 \quad$ Escape 3}

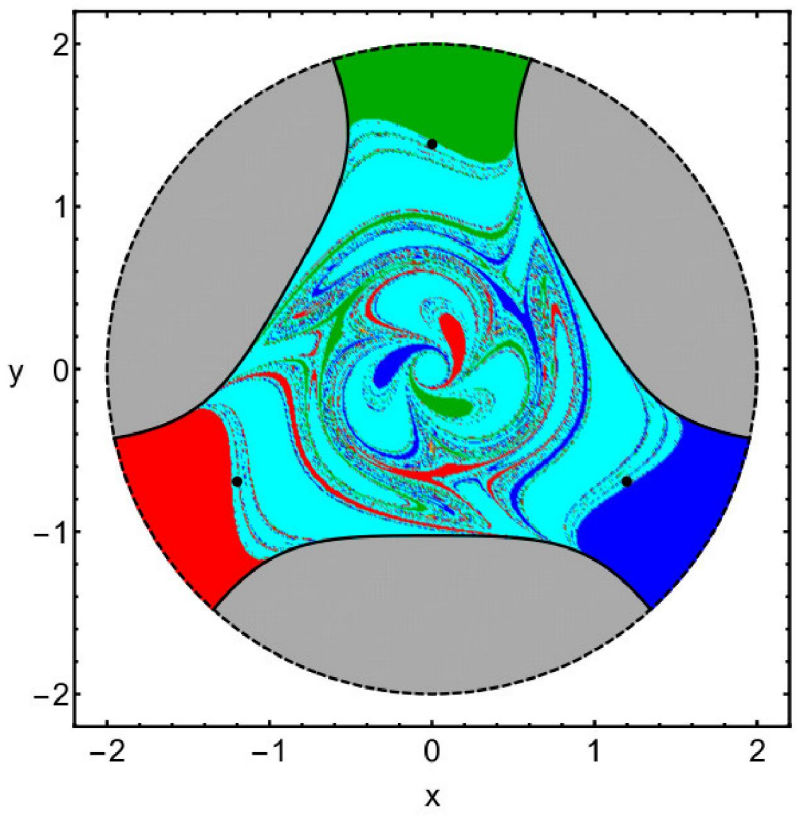

(d)

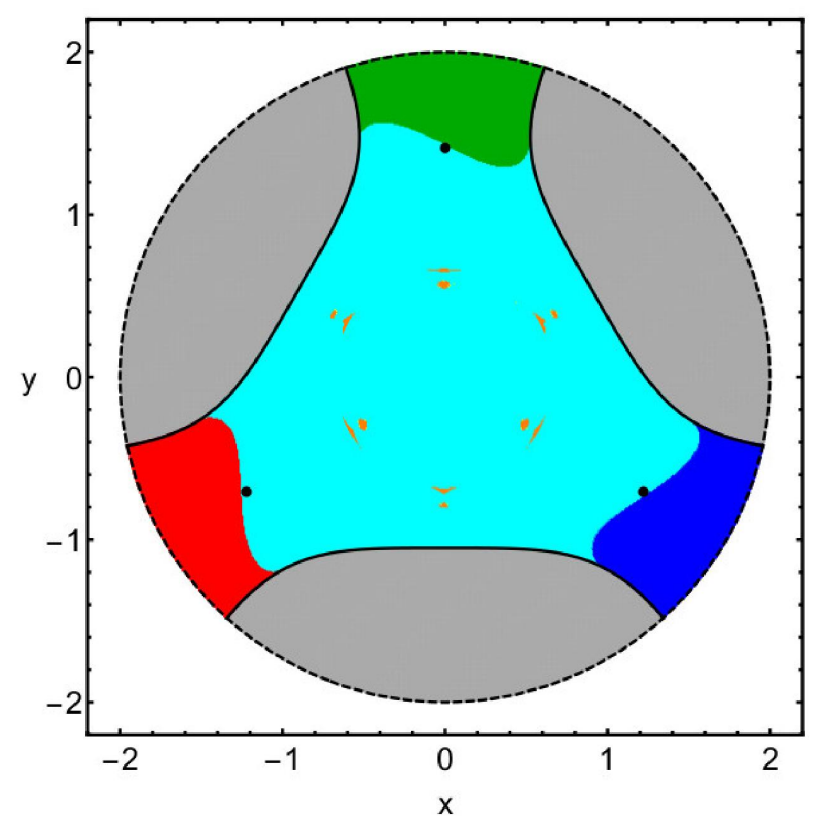

Regular Chaotic Collision

Fig. 7. Basin color diagrams on the plane $(x, y)$, for $E=-0.1$, when $(\mathrm{a}): \epsilon=0,(\mathrm{~b}): \epsilon=0.01,(\mathrm{c}): \epsilon=0.05,(\mathrm{~d}): \epsilon=0.1$. Black dots pinpoint the points of equilibrium. (Color figure online).

of bounded motion, we can further distinguish between ordered and chaotic motion, by deploying the SALI method [Skokos, 2001].

We begin by classifying trajectories with starting conditions on the plane $(x, y)$. Fig. 5 shows the nature of motion for four values of $\epsilon$, when $E=-0.5$. In part (a), where $\epsilon=0$ we observe that the most interesting orbital structure exists in the interior region, which is populated by all types of possible motion. 
(a)
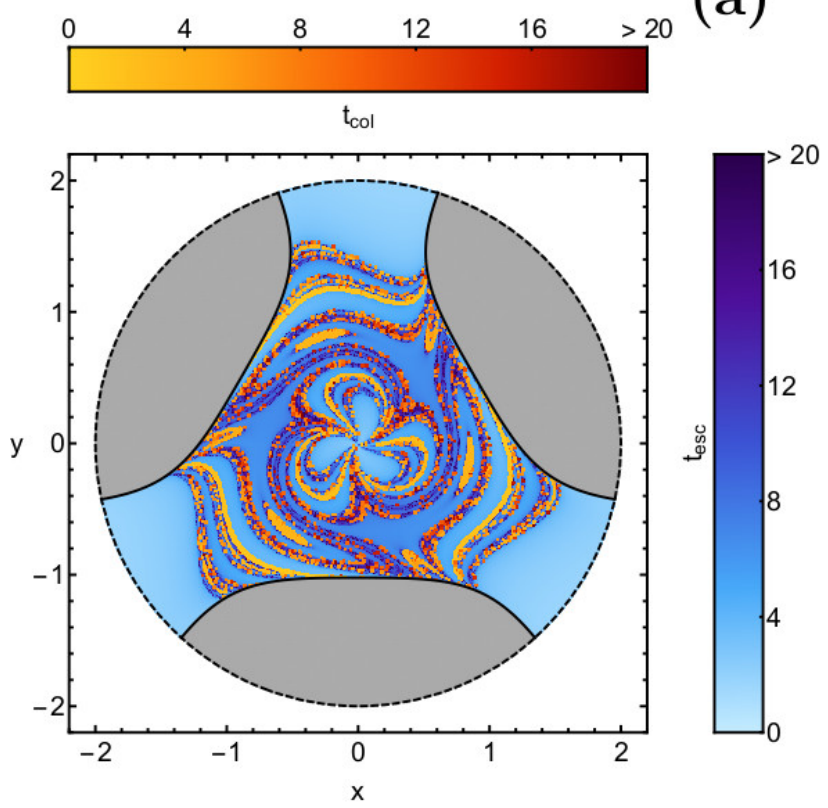

(c)

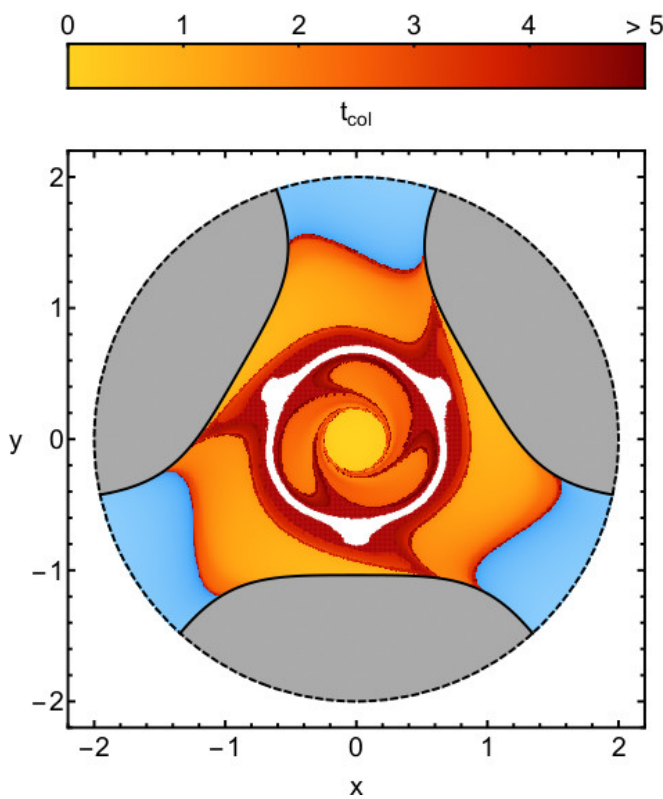

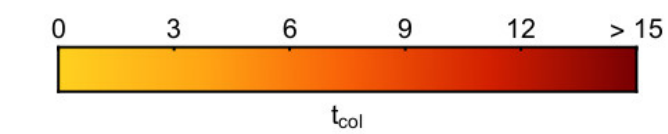

(b)
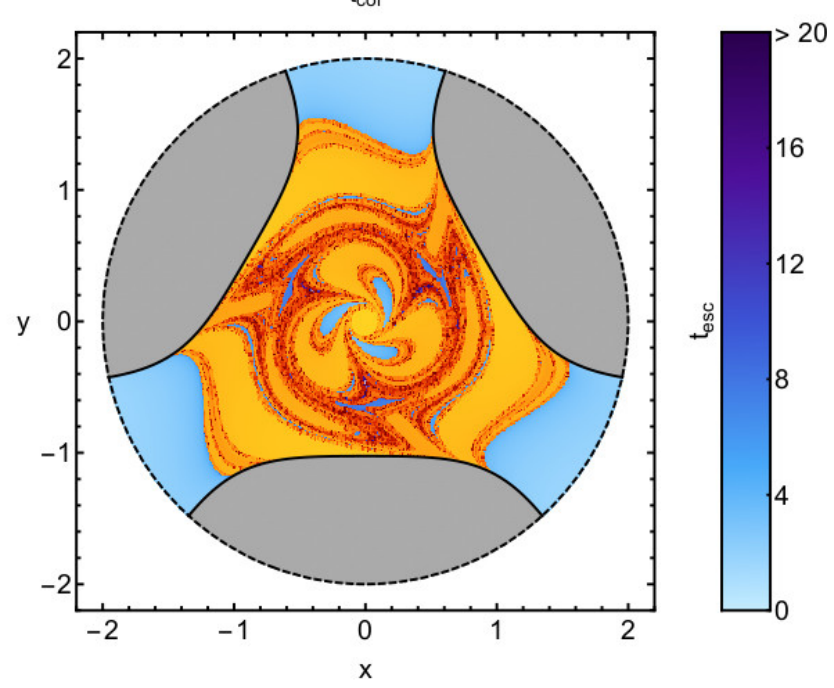

(d)

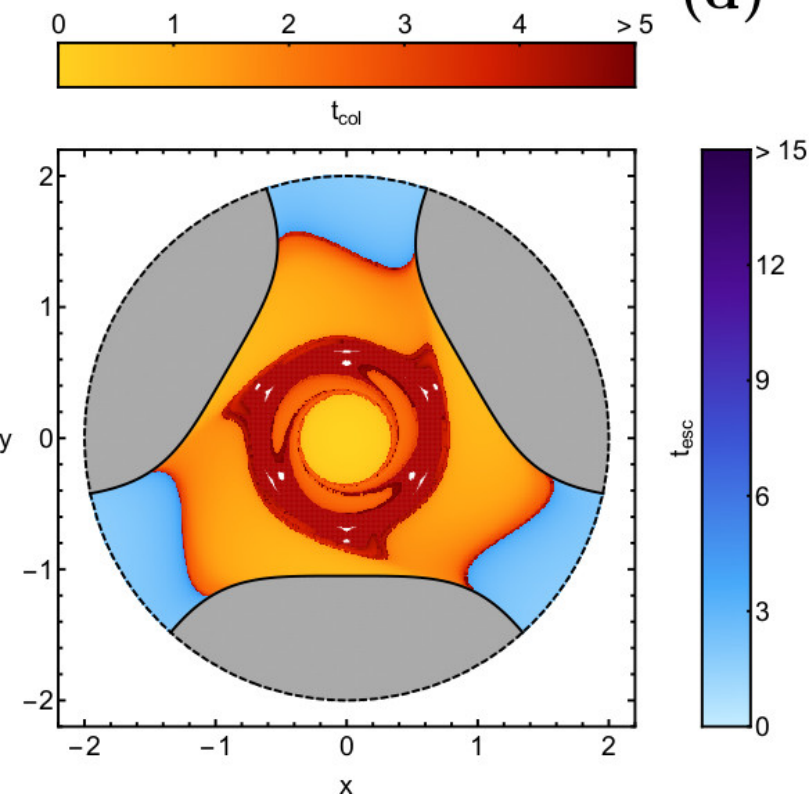

Fig. 8. Distributions of the collision and the escape time of the trajectories, for the initial conditions shown in parts (a)-(d) of Fig. 7. (Color figure online).

Moreover, in the interior region, one can find both basins of bounded, escaping or collision orbits, along with highly complicated areas with a fractal-like geometry. In panels (b)-(d) of Fig. 5 we see the influence of the transition parameter on the character of the orbits. The most noticeable phenomena that take place in the interior region are the following:

- The amount of collision orbits increases drastically and when $\epsilon \geq 0.1$ the entire interior region is dominated by initial conditions which lead to a collision with the singularity.

- The amount of both bounded and escaping orbits is reduced. In particular, escaping motion disappears more quickly that bounded motion.

- The fractal degree of the interior region is significantly reduced since all the fractal regions disappear, while well-formed basins of starting conditions (mainly basins of collision) dominate. 
(a)

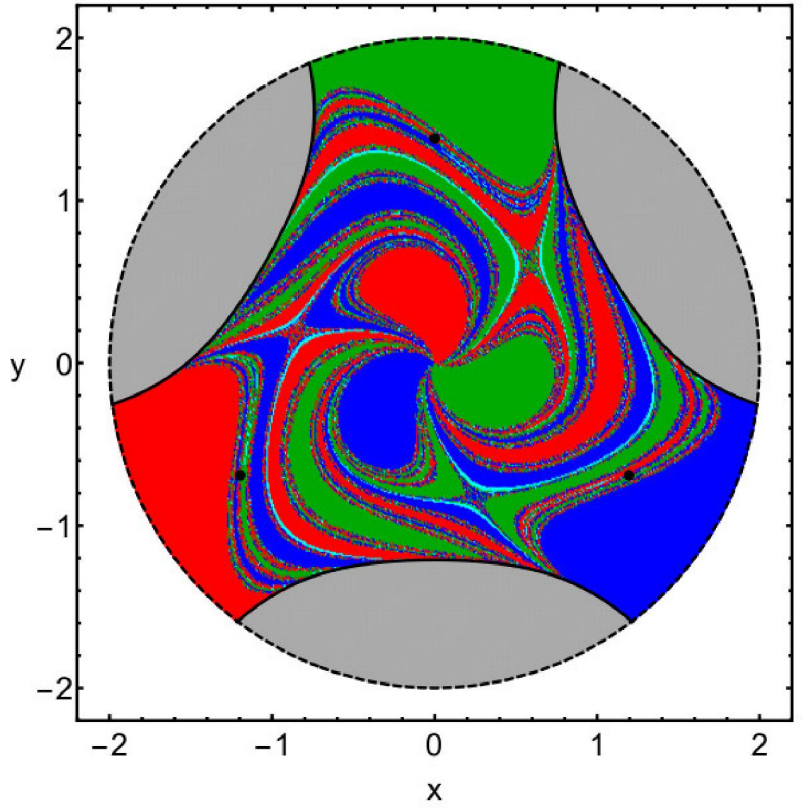

(c)

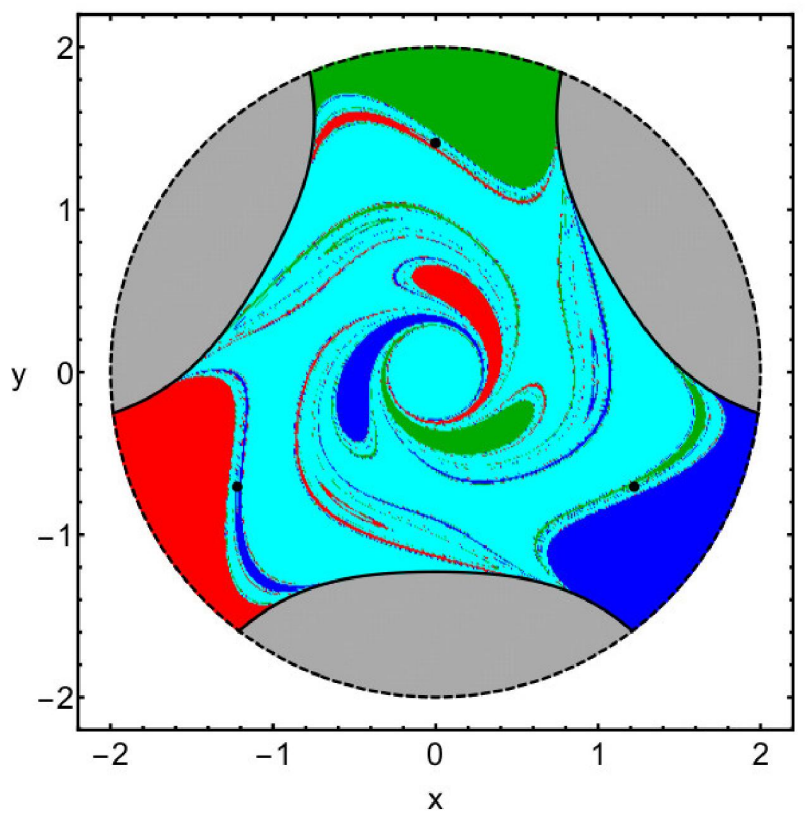

\section{Escape $1 \quad$ Escape 2 Escape 3}

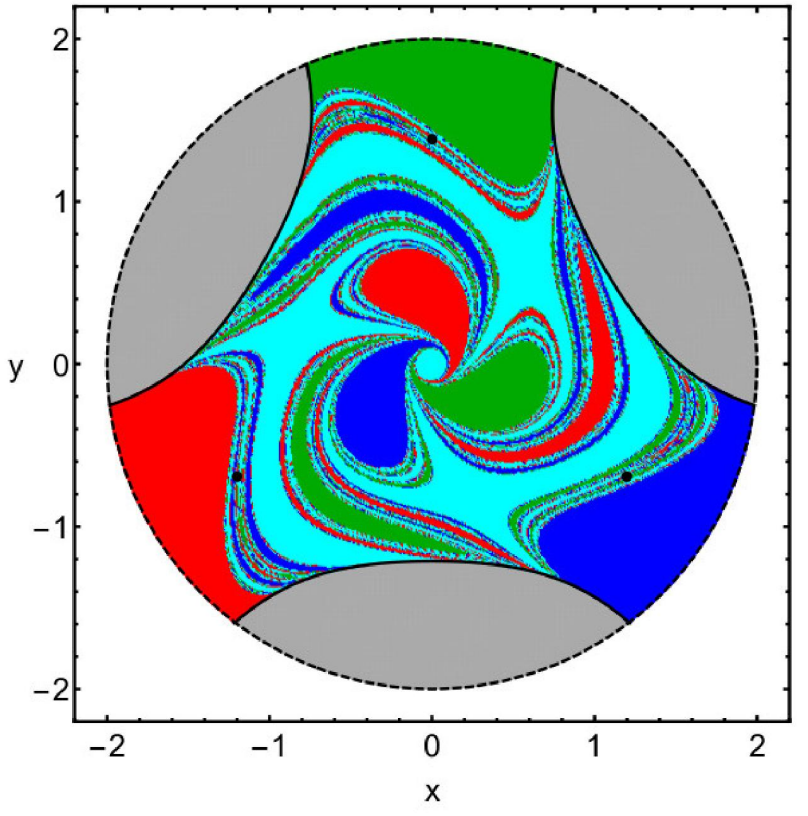

(d)

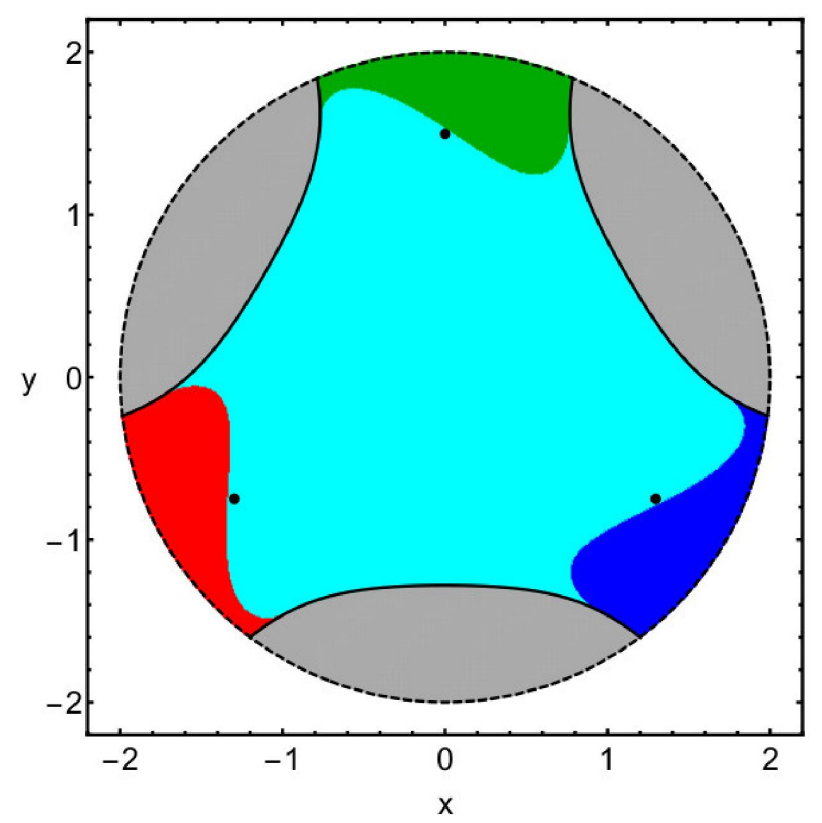

\section{$\begin{array}{llll}\text { Regular Chaotic Collision } & \end{array}$}

Fig. 9. Basin color diagrams on the plane $(x, y)$, for $E=0.5$, when $(\mathrm{a}): \epsilon=0,(\mathrm{~b}): \epsilon=0.01,(\mathrm{c}): \epsilon=0.1,(\mathrm{~d}): \epsilon=0.5$. Black dots pinpoint the points of equilibrium. (Color figure online).

In Fig. 6 we display how the respective escape and collision time of the trajectories are distributed on the plane $(x, y)$, when $E=-0.5$. It is evident that both the average escape and collision time of the trajectories are reduced, with increasing value of $\epsilon$.

Fig. 7 shows the character of the orbits on the plane $(x, y)$, when $E=-0.1$. Now, the orbital dynamics of the system, as a function of $\epsilon$, evolves similarly as in the case presented in Fig. 5. The only major 
(a)
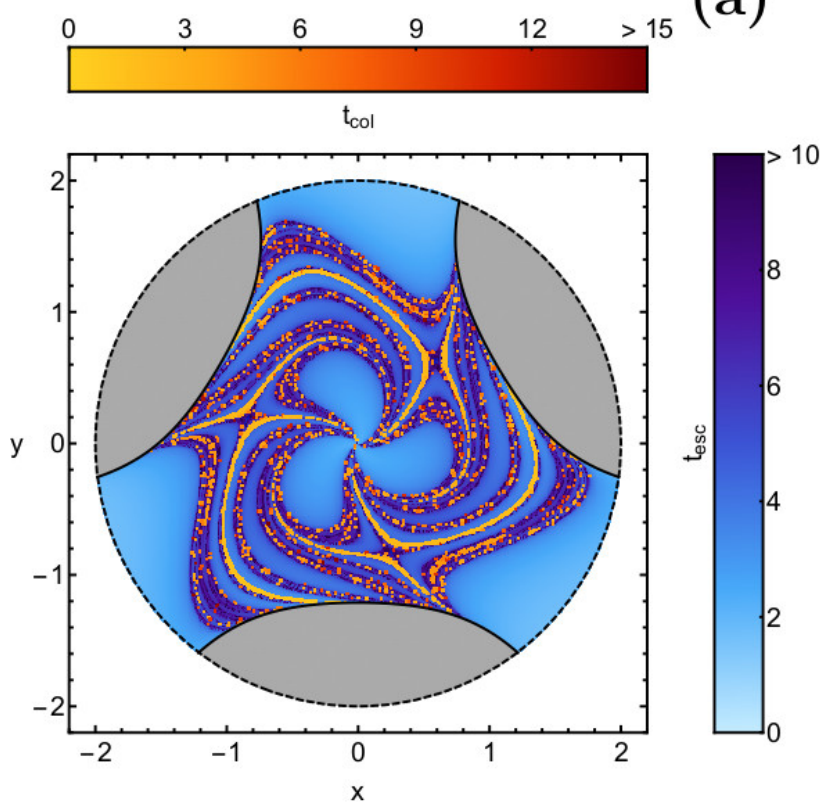

(c)

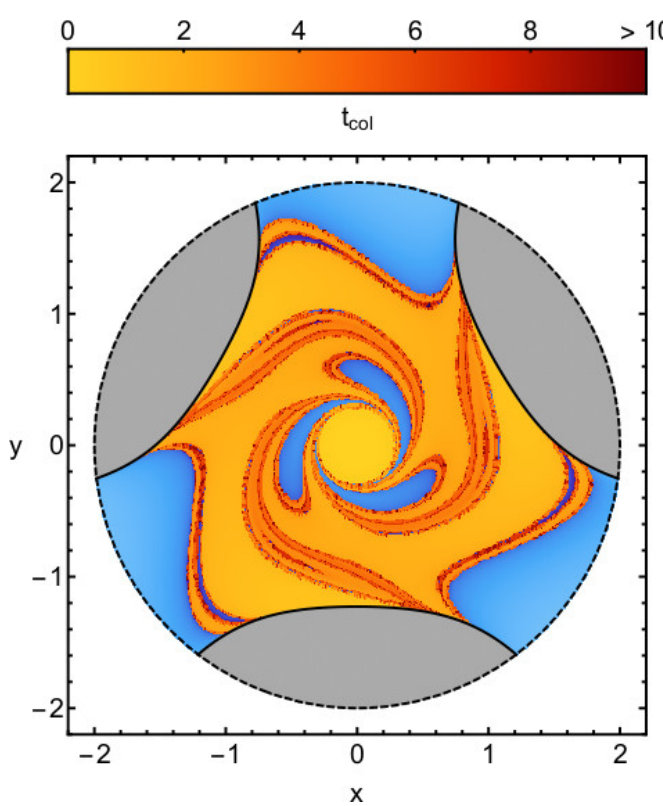

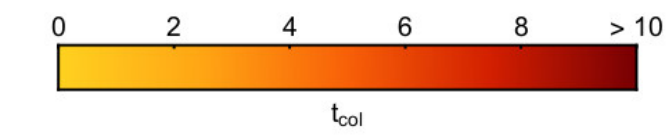

(b)
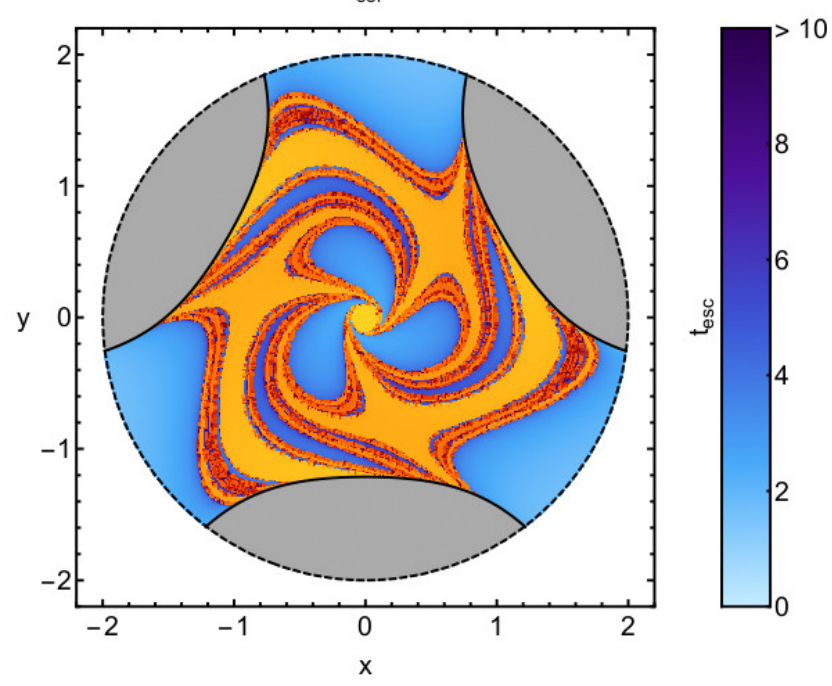

(d)
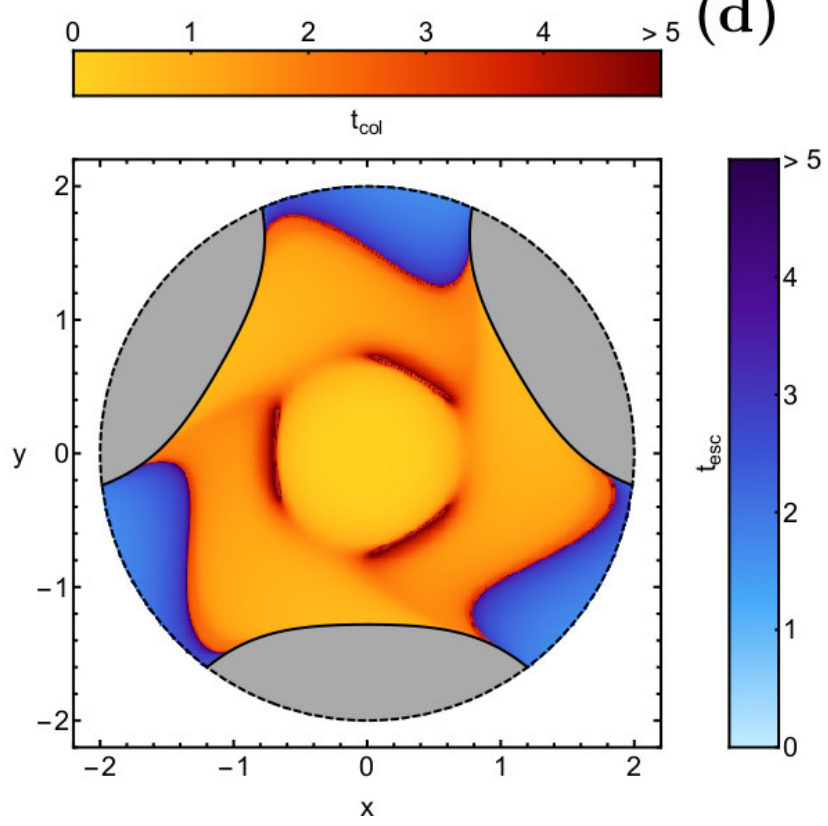

Fig. 10. Distributions of the collision and the escape time of the trajectories, for the initial conditions shown in parts (a)-(d) of Fig. 9. (Color figure online).

difference is the fact that non-escaping regular motion appears later, when $\epsilon=0.05$, while it remains present even when $\epsilon=0.1$. It should be pointed out that our analysis indicates that as the value of $\epsilon$ increases the stability basin splits into fragments before it disappears. The respective collision and escape time of the trajectories are given in the color diagrams of Fig. 8.

In the same vein in Fig. 9 we illustrate the orbital structure of the configuration plane when $E=0.5$. Once more, as $\epsilon$ increases the orbital structure of the interior region changes drastically. More specifically, the amount of collision trajectories grows rapidly, while escaping motion is reduced. However, for this energy level we observe an important difference, with respect to what we have seen earlier in Figs. 5 and 7. In particular, our computations suggest that for relatively high energy levels bounded motion is not possible, for any value of $\epsilon$. In parts (a)-(d) of Fig. 10 one can see how the collision and escape time of the 
(a)

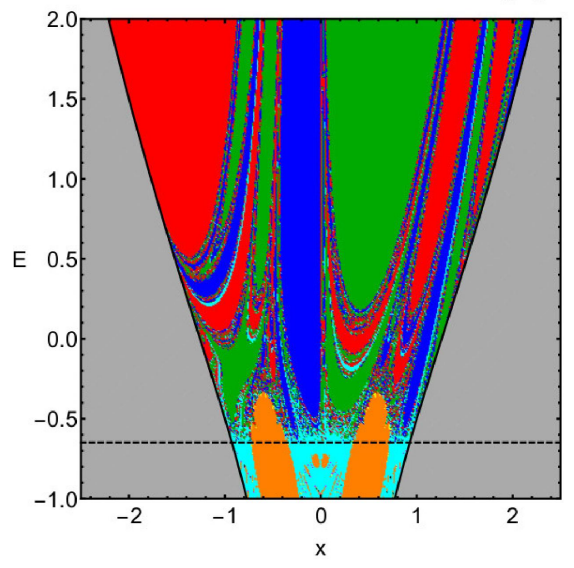

(c)

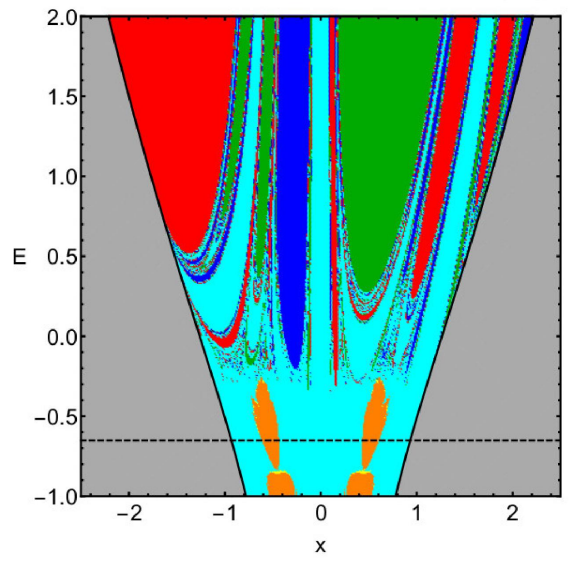

(e)

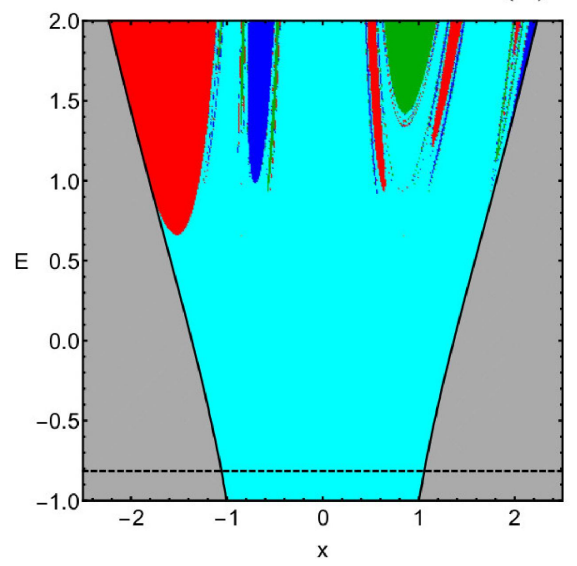

(b)

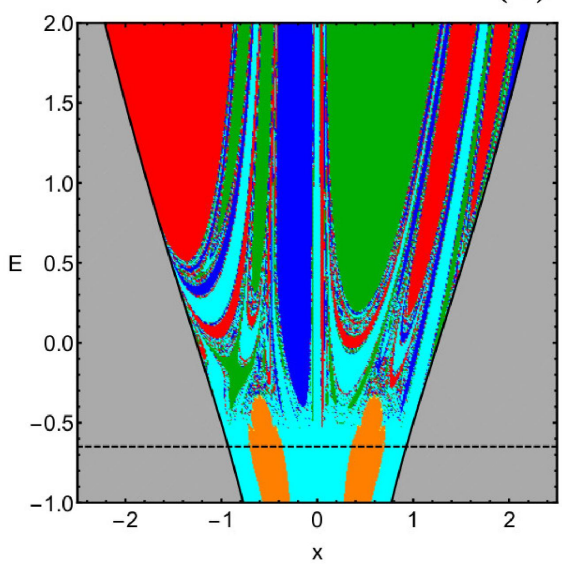

(d)

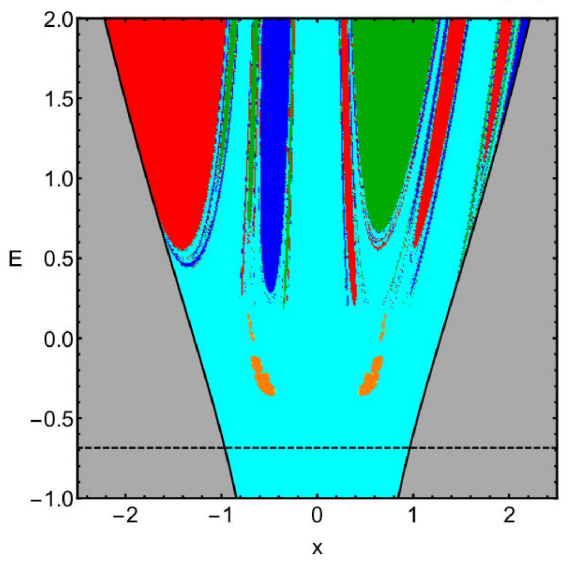

(f)

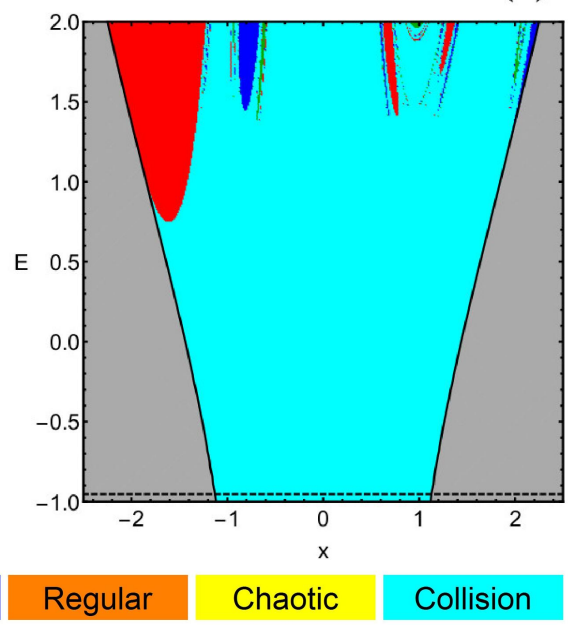

Fig. 11. Basin color diagrams on the $(x, E)$ plane, when (a): $\epsilon=0,(\mathrm{~b}): \epsilon=0.001,(\mathrm{c}): \epsilon=0.01,(\mathrm{~d}): \epsilon=0.1,(\mathrm{e}): \epsilon=0.5,(\mathrm{f})$ : $\epsilon=1$. The horizontal black dashes lines indicate the energy of escape. (Color figure online).

trajectories are distributed on the plane $(x, y)$, when $E=0.5$.

The basin diagrams of Figs. 5, 7, and 9 suggest that the basins of collision exist mostly in the interior region of the system, while they extend up to about the three saddle points. This implies that the central singularity has only a local effect. Moreover, even when $\epsilon=1$, that is when the term $1 / r^{3}$ has its full strength, the singularity located at $(0,0)$ cannot "attract" orbits launched outside the interior region, or 
(a)

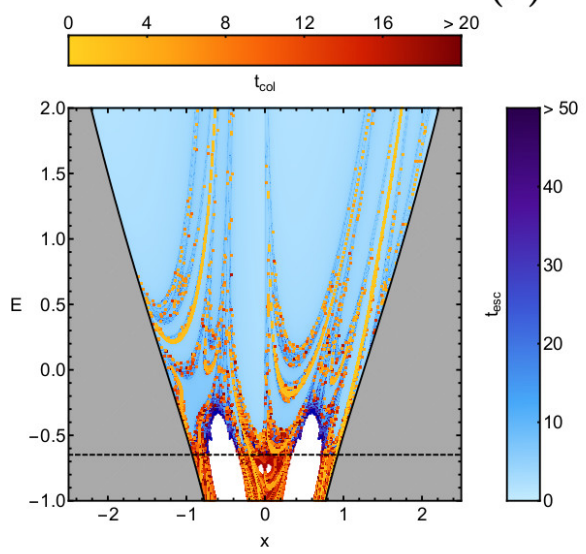

(c)

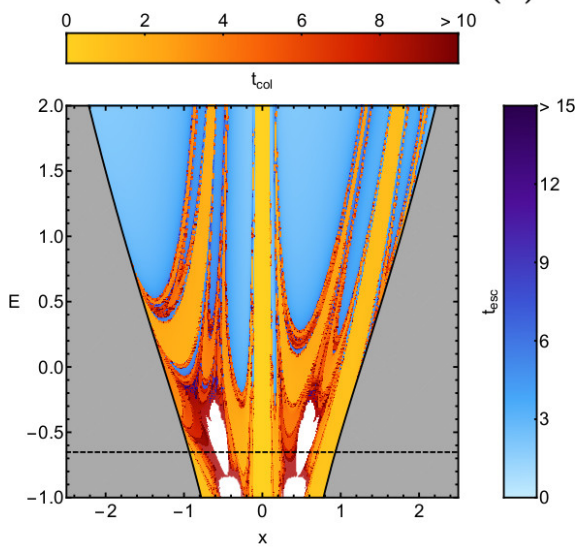

(e)

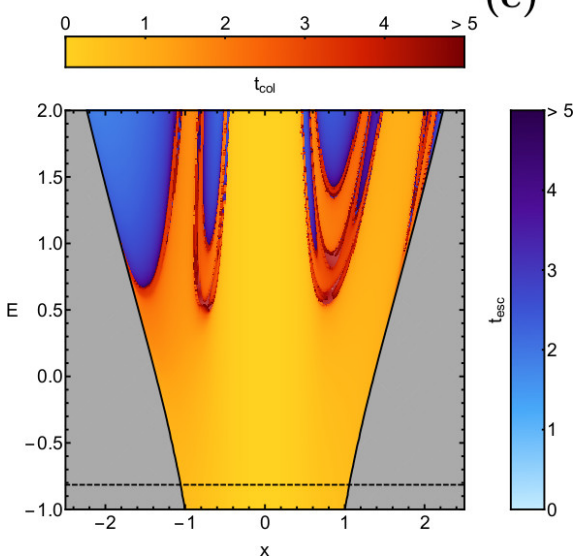

(b)

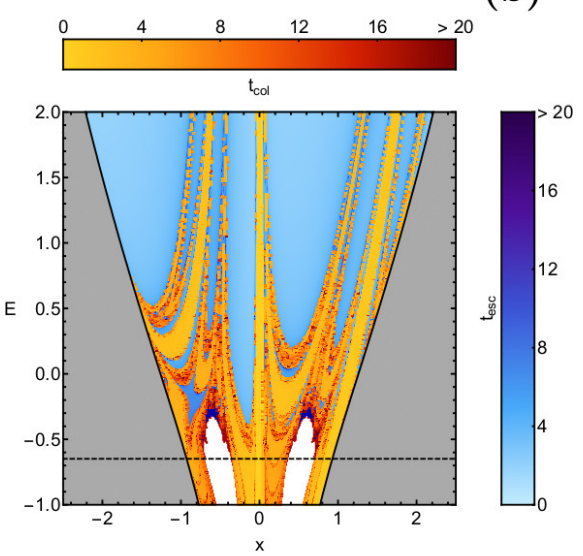

(d)

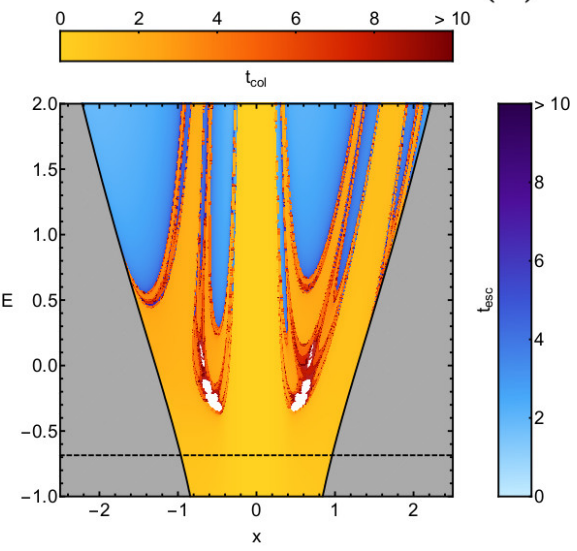

(f)

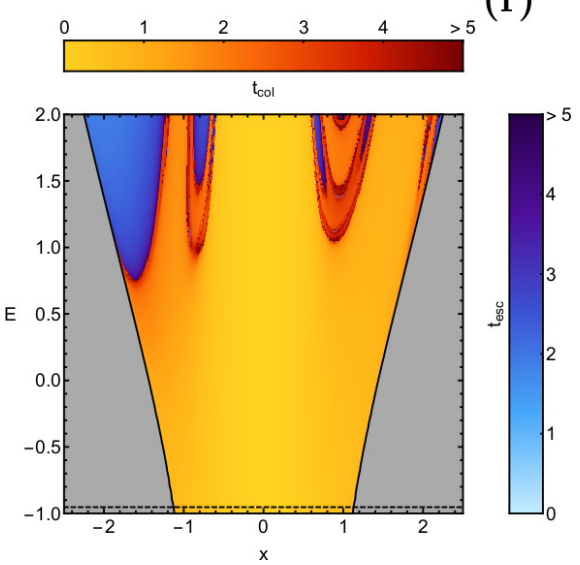

Fig. 12. Distributions of the collision and the escape time of the trajectories, for the initial conditions shown in parts (a)-(f) of Fig. 11. (Color figure online).

even coming from infinity.

More general information can be derived if we use a continuous spectrum of values of $E$. On this basis, in Fig. 11 we display the nature of motion, for several values of $\epsilon$, using initial conditions on the $(x, E)$ plane with $y_{0}=\dot{x_{0}}=0$, while $\dot{y_{0}}$ is always obtained through the second of equations (10). With increasing value of $\epsilon$ we observed the following changes:

- The basins of bounded regular orbits weaken and eventually, for relatively high values of $\epsilon(\epsilon>0.5)$ they completely disappear. 
(a)

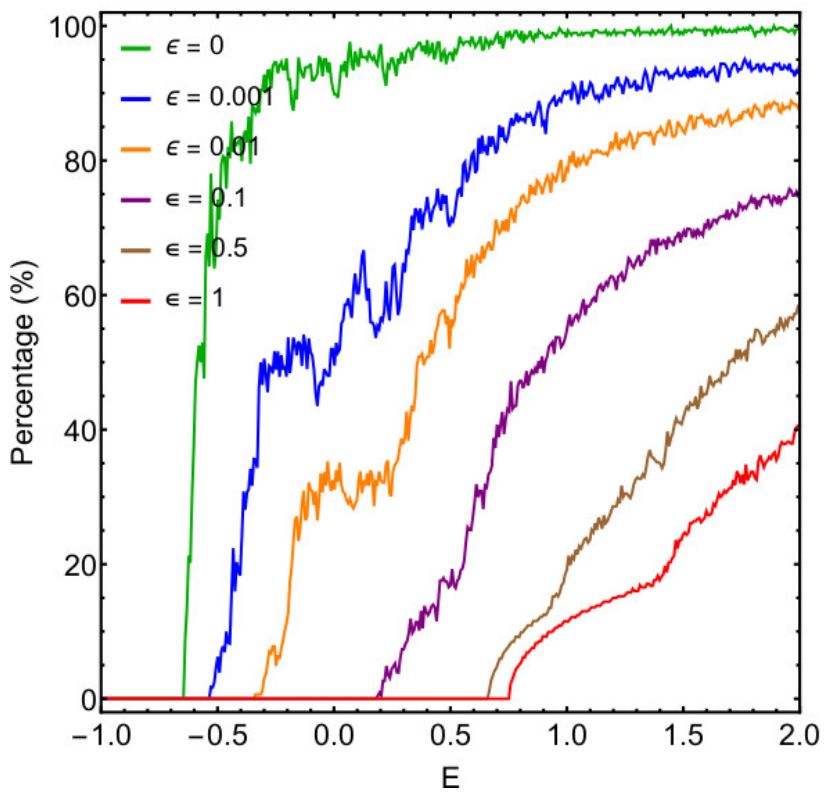

Fig. 13. Evolution of the percentage of (a): escape and (b): collision orbits on the $(x, E)$ plane, as a function of $E$. (Color figure online).

- The minimum value of the energy, at which escaping motion appears, increases.

- The area of the collision basins increases rapidly, while at the same time the fractal degree of the $(x, E)$ planes is reduced.

In Fig. 12 we provide diagrams with the corresponding distributions of the escape and collision time of the trajectories.

The evolution of the percentages of escape and collision starting conditions on the $(x, E)$ plane, as a function of $E$, is presented in parts (a)-(b) of Fig. 13. It is seen that with increasing $E$ the rate of escaping orbits grows, while that of collision motion is reduced. The transition parameter affects the parametric evolution as follows: escaping orbits appear later (for higher values of $E$ ), while their maximum value is reduced, as $\epsilon$ increases. As for the collision motion, the respective parametric evolution is entirely opposite, with respect to that of the escaping motion.

From the diagrams on the $(x, E)$ plane we can extract additional information, about the influence of the parameters $\epsilon$ and $E$ on the nature of motion in the interior region of the potential. Indeed, according to the plot shown in Fig. 14, the $(\epsilon, E)$ space can be divided into three main regions:

- Region $R_{1}$ : This region corresponds to energies below the energy of escape. Therefore, only bounded (chaotic and regular) and collision trajectories are possible.

- Region $R_{2}$ : There all three main types of motion (bounded, escaping and collision) are possible.

- Region $R_{3}$ : In this region collision motion completely dominates, while bounded and escaping types of motion are not possible.

Going one step further we can also deduce useful results by classifying trajectories with starting conditions on the $(x, \epsilon)$ plane. In Fig. 15 we show the character of motion for four values of $E$. Inspecting the basin diagrams in parts (a)-(d) of Fig. 15 it becomes evident that for low values of the energy collision motion dominates, while as the value of the energy increases the area of the basins of escape grows significantly and covers the majority of the $(x, \epsilon)$ space. Once more, it is confirmed that regular bounded motion exists only for relatively low values of $E$. The respective distributions of the collision and escape time of the trajectories are given in parts (a)-(d) of Fig. 16. One can see that both the average values of the phenomena of escape and collision are reduced with increasing $E$. On the other hand, we are not in a 


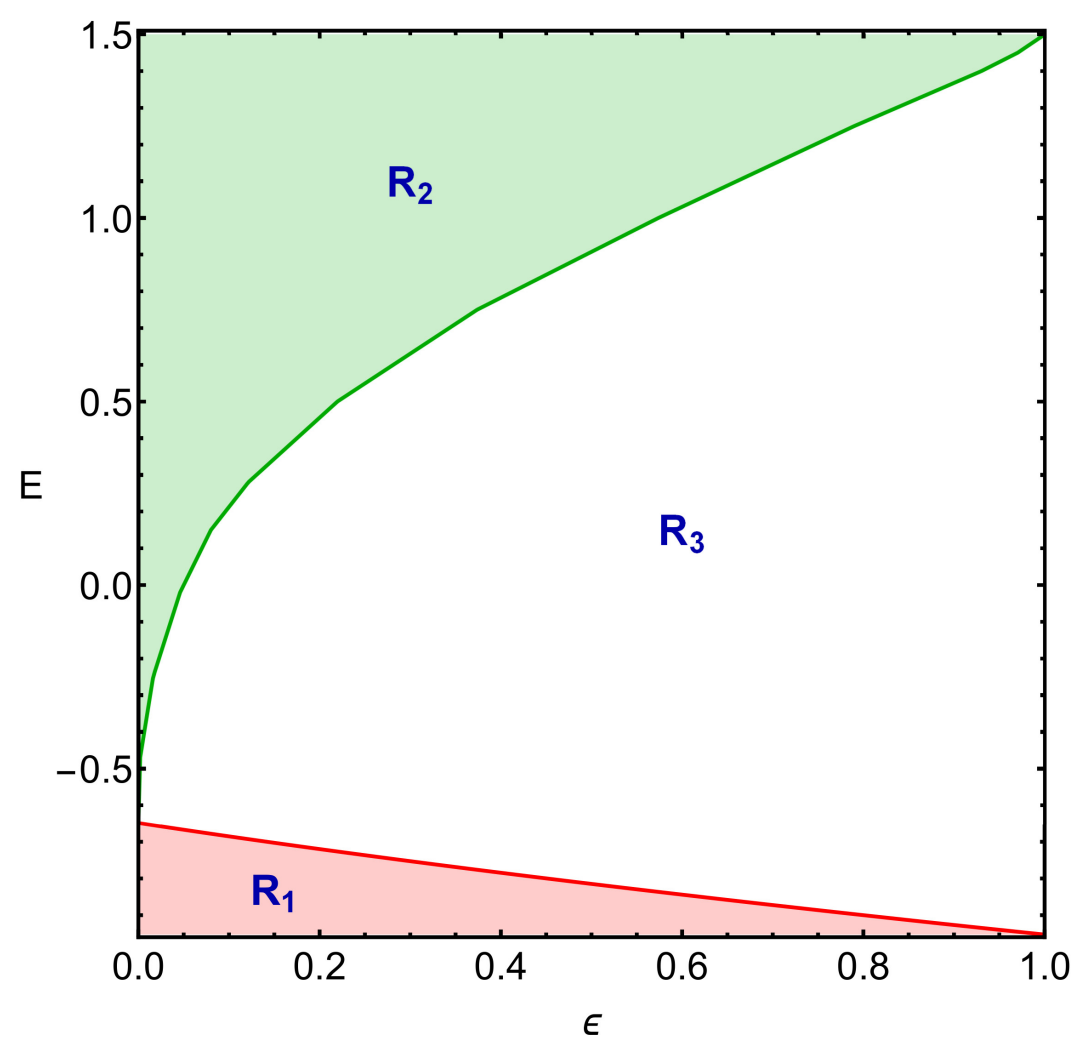

Fig. 14. Regions of possible types of motion on the $(\epsilon, E)$ space. More details are given in the text. The solid red line corresponds to the parametric evolution of the energy of escape. (Color figure online).

position to extract safe conclusions regarding the effect of the transition parameter on the time scale of the phenomena. Our calculations suggest that for all tested energy levels, the average values of the collision and escape time of the trajectories fluctuate, as $\epsilon$ varies in the interval [0,1], without exhibiting any clear pattern of increase or decrease.

\section{Concluding remarks}

This article was devoted to determining the character of motion in a new version of the classical HénonHeiles system, where an additional gravitational potential is added, for modeling the dynamics of the central singularity. For revealing the dynamics of the trajectories of the massless particle, we numerically integrated vast sets of starting conditions in different types of $2 \mathrm{D}$ planes. Additionally, for illustrating the different types of basins, we deployed modern diagrams with color codes, which helped us to unveil how the energy, as well as the transition parameter, affect the final state of the trajectories.

The following list captures the most interesting outcomes of our analysis:

(1) Always the three points of equilibrium are linearly unstable when $\epsilon \in[0,1]$.

(2) As $\epsilon$ increases the area of the collision basins grows significantly, while at the same time the extent of both the bounded basins and the basins of escape is reduced.

(3) Bounded motion mainly exists for low values of the transition parameter and the energy.

(4) The basins of escape of the system extend to infinity, while on the contrary, the basins of collision are mainly present only up to the three saddle points.

(5) Both the average values of the collision and escape time of the trajectories are reduced, as the value of the energy increases. On the other hand, the transition parameter seems not to have a clear influence on the evolution of the time scales of the phenomena.

All the starting conditions of the trajectories were numerically integrated by using a Bulirsch-Stoer routine written in FORTRAN 77[Press et al., 1992], with double precision, which ensured that the Hamilto- 
(a)

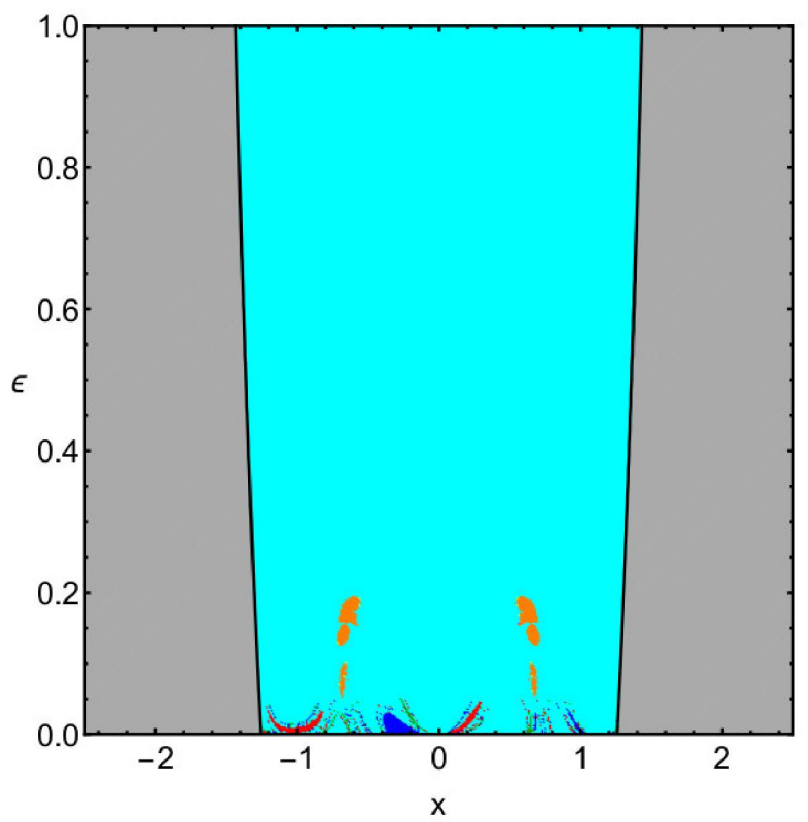

(c)

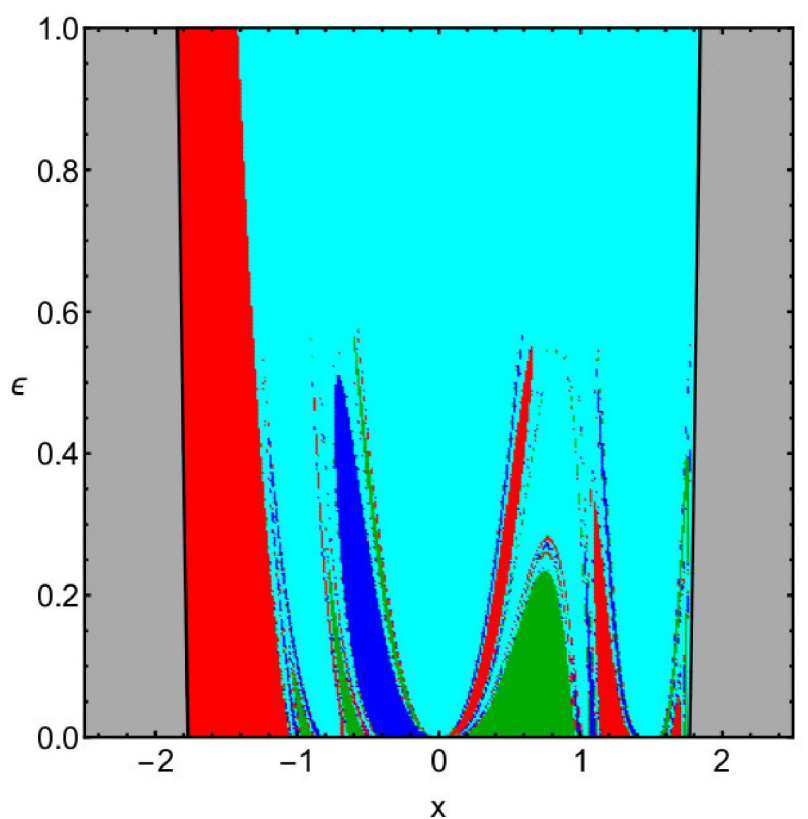

\section{Escape $1 \quad$ Escape 2 Escape 3}

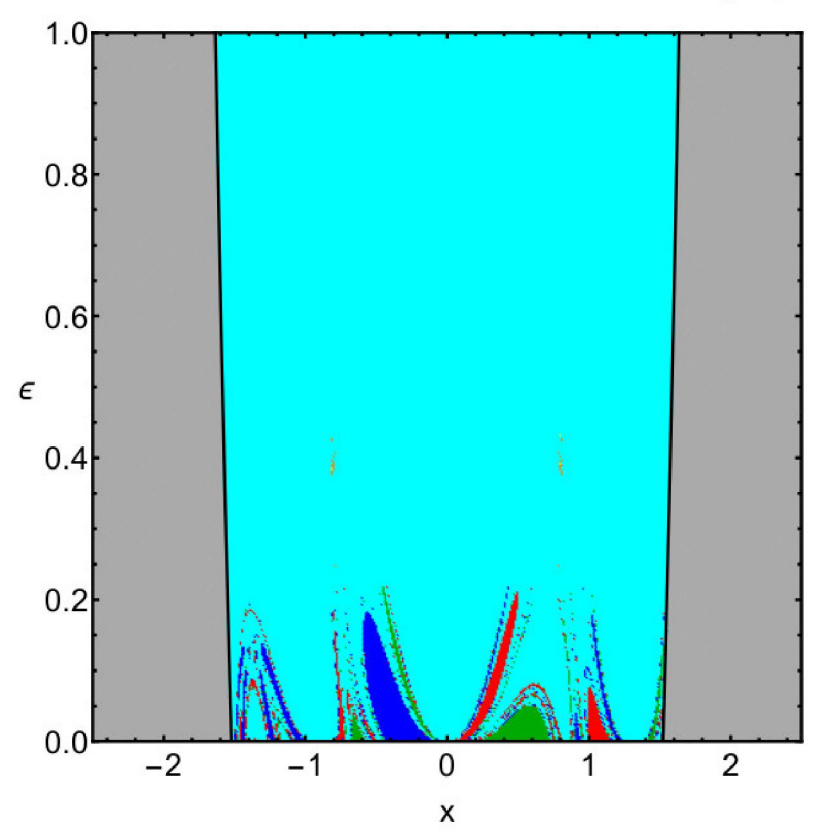

(d)

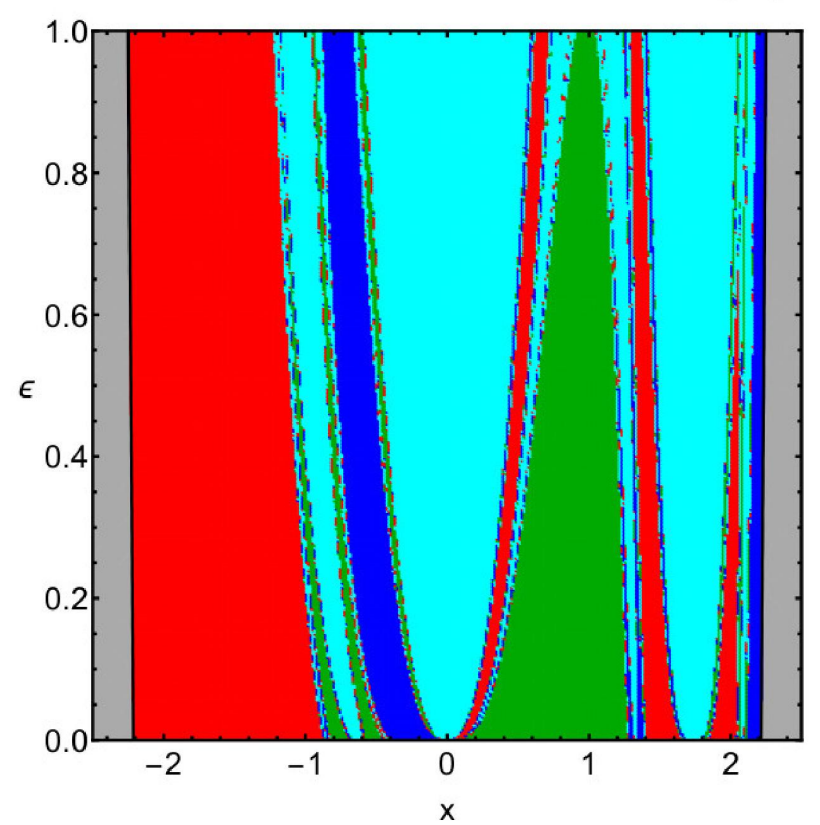

\section{Regular Chaotic Collision}

Fig. 15. Basin color diagrams on the $(x, \epsilon)$ plane, when (a): $E=-0.01,(\mathrm{~b}): E=0.5,(\mathrm{c}): E=1,(\mathrm{~d}): E=2$. (Color figure online).

nian was sufficiently conserved, while the relative error was of the order of $10^{-14}$, or lower. The required computation time, per set of starting conditions, was varying between 0.5 and 12 hours, using a Quad-Core i7 4.0 GHz CPU. The latest version 12.0 of Mathematica ${ }^{\circledR}[$ Wolfram, 2003] was used for creating all the graphics of the article. 
(a)
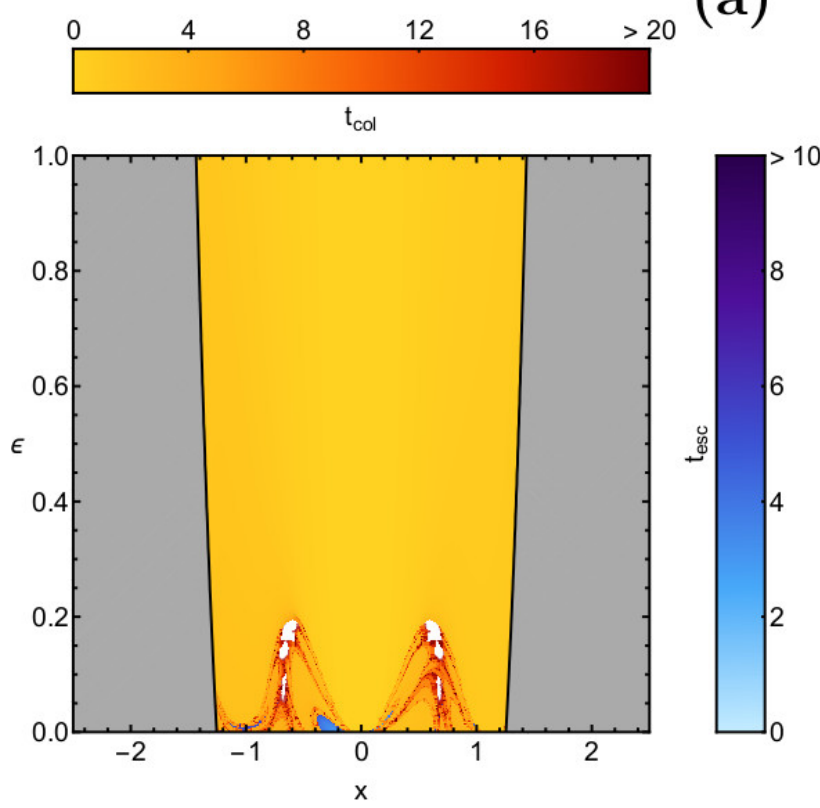

(c)
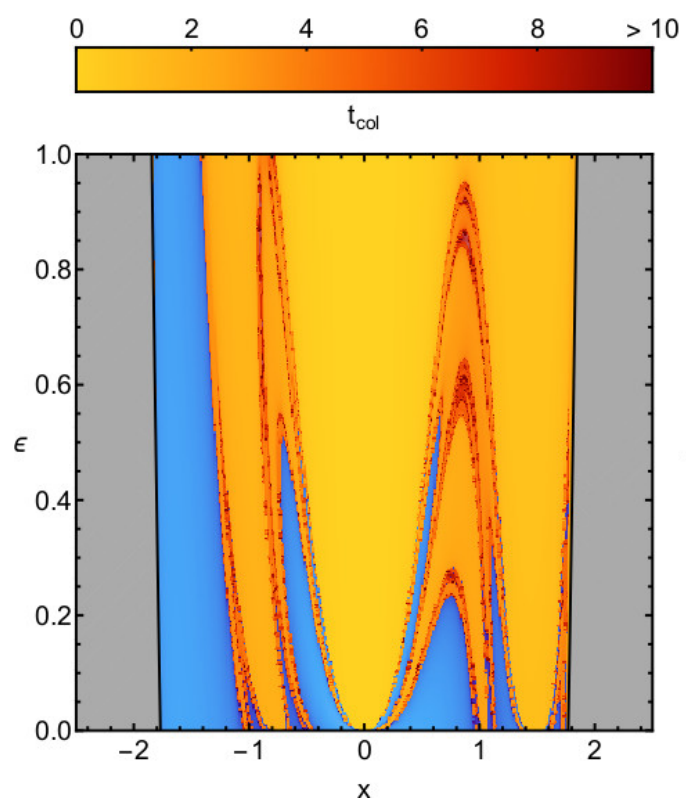

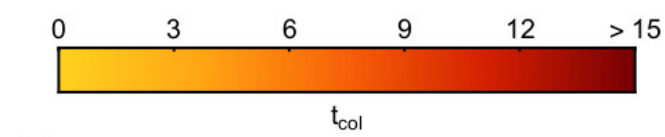

(b)

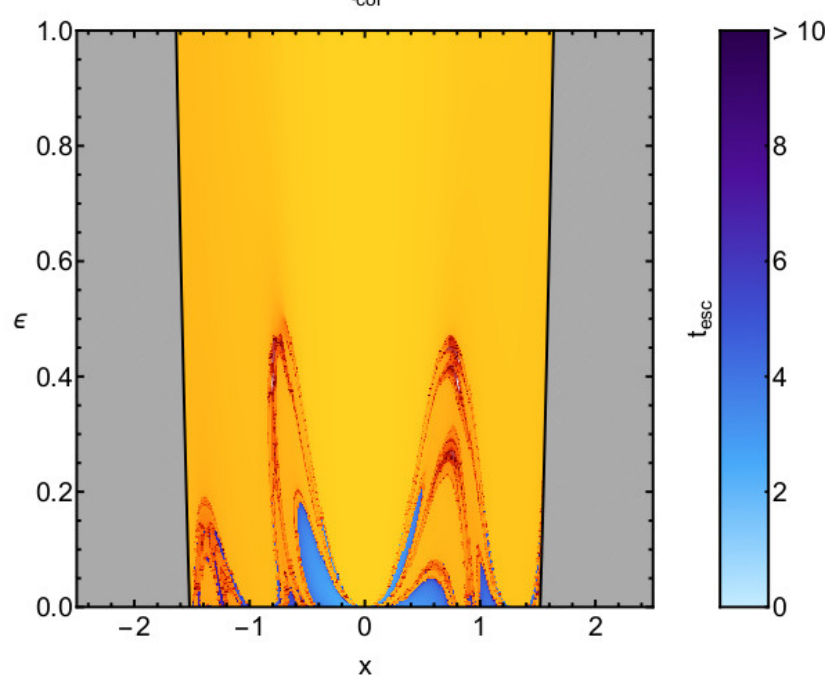

(d)

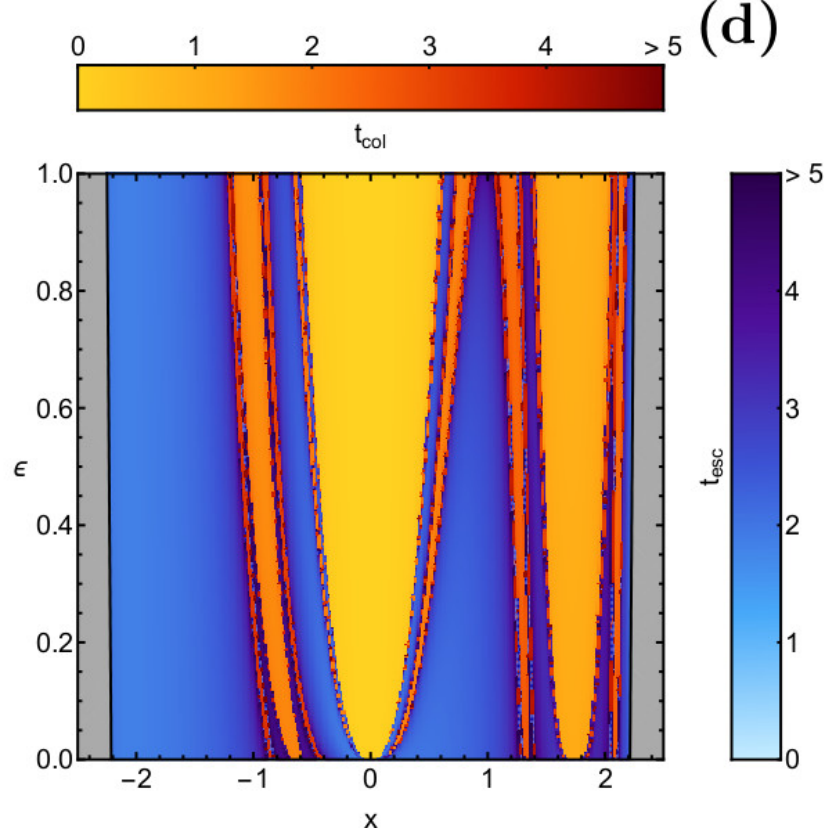

Fig. 16. Distributions of the collision and the escape time of the trajectories, for the initial conditions shown in parts (a)-(f) of Fig. 15. (Color figure online).

\section{References}

Aguirre, J., Vallejo, J.C. and Sanjuán, M.A.F. [2001] "Wada basins and chaotic invariant sets in the Hénon-Heiles system", Phys. Rev. E 64, 066208.

Aguirre J. and Sanjuán M.A.F. [2003a] "Limit of small exits in open Hamiltonian systems". Phys. Rev. E 67, 056201-1056201-7.

Aguirre, J., Vallejo, J.C. and Sanjuán, M.A.F. [2003b] "Wada basins and unpredictability in Hamiltonian and dissipative systems". Int. J. Mod. Phys. B 17, pp. 4171-4175.

Aguirre, J., Viana, R.L. and Sanjuán, M.A.F. [2009] "Fractal structures in nonlinear dynamics", Rev. Mod. Phys. 81, pp. 333-386.

Barrio, R., Blesa, F. and Serrano, S. [2008] "Fractal structures in the Hénon-Heiles Hamiltonian". Europhys. Lett. 82, 100031-10003-6. 
Blesa, F., Seoane, J.M., Barrio, R. and Sanjuán, M.A.F. [2012] "To escape or not to escape, that is the question - Perturbing the Hénon-Heiles Hamiltonian". Int. J. Bifurc. Chaos 22, 1230010-1-1230010-9.

Brack, M. [2001] "Orbits with analytical scaling constants in Hénon-Heiles type potentials". Fundations of Phys. 31, pp. 209-232.

Chang, Y.F., Tabor, M. and Weiss, J. [1982] "Analytic structure of the Hénon-Heiles Hamiltonian in integrable and nonintegrable regimes". J. Math. Phys. 23, pp. 531-538.

Churchill, R., Pecelli. G. and Rod, D.L. [1975] "Isolated unstable periodic orbits". J. Differ. Equ. 17, pp. 329-348.

Coccolo, M., Seoane, J.M. and Sanjuán, M.A.F. [2013] "Controlling unpredictability in the randomly driven Hénon-Heiles system". Communications in Nonlinear Science and Numerical Simulation 18, pp. 3449-3457.

Conte, R., Musette, M. and Verhoeven, C. [2005] "Explicit integration of the Hénon-Heiles Hamiltonians". Journal of Nonlinear Mathematical Physics 12, pp. 212-227.

De Moura, A.P.S. and Letelier, P.S. [1999] "Fractal basins in Hénon-Heiles and other polynomial potentials". Physics Letters A 256, pp. 362-368.

Deprit, A. and Henrard, J. [1968] "A manifold of periodic orbits". Adv. in Astron. and Astrophys. 6, pp. 1-124.

Deprit, A. and Henrard, J. [1969] "Construction of orbits asymptotic to a periodic orbit"". Astron. J. 74, pp. 308-316.

Dubeibe, F.L., Lora-Clavijo, F.D. and Guillermo, A.G. [2017a] "Pseudo-Newtonian planar circular restricted 3-body problem". Phys. Let. A 381, pp. 563-567.

Dubeibe, F.L., Lora-Clavijo, F.D. and Guillermo, A.G. [2017b] "On the conservation of the Jacobi integral in the postNewtonian circular restricted three-body problem". Astrophys. Space Science 362, 97.

Fordy, A.P. [1991] "The Hénon-Heiles system revisited". Phys. D: Nonlinear Phenomena 52, pp. 204-210.

Hénon, M. and Heiles, C. [1964] "The applicability of the third integral of motion: some numerical experiments". Astron. J. 69, pp. 73-79.

Innanen, K.A. [1985] "The threshold of chaos for Hénon-Heiles and related potentials". Astron. J. 90, pp. 2377-2380.

Lyapunov, A.M. [1907] "Problème général de la stabilité du movement". Ann. Fac. Sci. Univ. Toulouse 9, pp. $203-475$.

Nagler, J. [2004] "Crash test for the Copenhagen problem". Phys. Rev. E 69, 066218.

Nagler, J. [2005] "Crash test for the restricted three-body problem". Phys. Rev. E 71, 026227.

Ozaki, J. and Kurosaki, S. [1996] "Periodic orbits of Hénon-Heiles Hamiltonian". Progress of Theoretical Physics 95, pp. 519-529.

Plummer, H.C. [1991] "On the problem of distribution in globular star clusters". MNRAS 71, pp. 460-470.

Poincaré, H. [1892] "Les Méthodes nouvelles de la mécanique céleste", Gauthier-Villars, Paris.

Press, H.P., Teukolsky, S.A., Vetterling, W.T. and Flannery, B.P. [1992] "Numerical Recipes in FORTRAN 77", 2nd edn. Cambridge University Press, Cambridge, USA.

Schwarzschild, K. [1916] "Über das Gravitationsfeld eines Massenpunktes nach der Einstein'schen Theorie". Sitzungsberichte der Königlich Preussischen Akademie der Wissenschaften 1, pp 189-196.

Skokos, C. [2001] "Alignment indices: A new, simple method for determining the ordered or chaotic nature of orbits". J. Phys. A: Math. Gen. 34, pp. 10029-10043.

Suraj, Md S., Zotos, E.E., Aggarwal, R. and Mittal, A. [2019] "Unveiling the basins of convergence in the pseudo-Newtonian planar circular restricted four-body problem". New Astronomy 66, pp. 52-67.

Wolfram, S. [2003] "The Mathematica Book". Wolfram Media, Champaign.

Zotos, E.E. [2015] "Classifying orbits in the classical Hénon-Heiles Hamiltonian system". Nonlinear Dyn. 79, pp. $1665-1677$.

Zotos, E.E. [2017a] "An overview of the escape dynamics in the Hénon-Heiles Hamiltonian system". Meccanica 52, pp. $2615-2630$.

Zotos, E.E. [2017b] "Basins of convergence of equilibrium points in the pseudo-Newtonian planar circular restricted three-body problem". Astrophys. Space Science 362, 195.

Zotos, E.E., Dubeibe, F.L. and Guillermo, A.G. [2018] "Orbit classification in an equal-mass non-spinning binary black hole pseudo-Newtonian system". MNRAS 477, pp. 5388-5405.

Zotos, E.E., Dubeibe, F.L., Nagler, J. and Tejeda, E. [2019] "Orbit classification in a pseudo-Newtonian Copenhagen problem with Schwarzschild-like primaries". MNRAS 487, pp. 2340-2353. 